\title{
A Dual-Polarization Radar Synthetic QPE for Operations
}

\author{
JiAn ZhANG, ${ }^{\mathrm{a}}$ Lin TANG, ${ }^{\mathrm{a}, \mathrm{b}}$ STEPHEN COCKs,,${ }^{\mathrm{a}, \mathrm{b}}$ PENGFEI ZHANG, ${ }^{\mathrm{a}, \mathrm{b}}$ AlEXANDER RyZHKOV,, a,b \\ Kenneth Howard,${ }^{a}$ CARrie LANGSTON, ${ }^{a, b}$ AND BRIAN KANEY ${ }^{a, b}$ \\ ${ }^{\text {a }}$ NOAA/OAR/National Severe Storms Laboratory, Norman, Oklahoma; ${ }^{\mathrm{b}}$ Cooperative Institute for Mesoscale Meteorological \\ Studies, University of Oklahoma, Norman, Oklahoma
}

(Manuscript received 26 August 2019, in final form 22 January 2020)

\begin{abstract}
A new dual-polarization (DP) radar synthetic quantitative precipitation estimation (QPE) product was developed using a combination of specific attenuation $A$, specific differential phase $K_{\mathrm{DP}}$, and reflectivity $Z$ to calculate the precipitation rate $R$. Specific attenuation has advantages of being insensitive to systematic biases in $Z$ and differential reflectivity $Z_{\mathrm{DR}}$ due to partial beam blockage, attenuation, and calibration while more linearly related to $R$ than other radar variables. However, the $R(A)$ relationship is not applicable in areas containing ice. Therefore, the new DP QPE applies $R(A)$ in areas where radar is observing pure rain, $R\left(K_{\mathrm{DP}}\right)$ in regions potentially containing hail, and $R(Z)$ elsewhere. Further, an evaporation correction was applied to minimize false light precipitation related to virga. The new DP QPE was evaluated in real time over the conterminous United States and showed significant improvements over previous radar QPE techniques that were based solely on $R(Z)$ relationships. The improvements included reduced dry biases in heavy to extreme precipitation during the warm season. The new DP QPE also reduced errors and spatial discontinuities in regions impacted by partial beam blockage. Further, the new DP QPE reduced wet bias for scattered light precipitation in the southwest and north central United States where there is significant boundary layer evaporation.
\end{abstract}

KEYWORDS: Freshwater; Precipitation; Hydrometeorology; Radars/Radar observations; Remote sensing; Flood events

\section{Introduction}

High-resolution and high-accuracy quantitative precipitation estimation (QPE) is a key component for many applications in agriculture, manufacturing, flash flood detection, river flood prediction, water resource managements, and climate assessments. The observation of precipitation requires various remote sensing systems and networks encompassing surface rain/snow gauges, radar, and satellite. Among them, ground radar networks currently provide the highest spatial and temporal resolution QPEs that are a critical input for flash flood warnings.

Beginning in the 1990s, the National Weather Service Weather Surveillance Radar-1988 Doppler (WSR-88D) network has been producing an operational radar QPE identified as the Precipitation Process System (PPS; Fulton et al. 1998). While PPS provided high-resolution precipitation distributions in areas that were lacking in gauge observations, it suffered contamination from nonmeteorological returns especially in spring and fall seasons when anomalous propagations (AP) and biological echoes (so-called "blooms") were most pronounced. The WSR-88D network was upgraded to dualpolarization (DP) in 2011-13, which included a near surface hydrometeor classification (HC; Park et al. 2009) and a DP radar QPE (Giangrande and Ryzhkov 2008) algorithm implemented at each radar site. The $\mathrm{HC}$ was based on fuzzy logic principles and membership functions utilizing all DP radar variables. The advancements in $\mathrm{HC}$ provided a much-improved identification of nonhydrometeor returns over the singlepolarization (SP) radar techniques. Subsequently, the DP

Corresponding author: Jian Zhang, jian.zhang@noaa.gov
QPE (also called "DPR" for digital precipitation rate; https:/training.weather.gov/wdtd/courses/dualpol/documents/ DualPolRadarPrinciples.pdf) had less contamination from anomalous propagation clutter and biological scatters than PPS. The DPR QPE, based on reflectivity $Z$, differential reflectivity $Z_{\mathrm{DR}}$, and specific differential phase $K_{\mathrm{DP}}$, provided improved precipitation estimates (less mean bias) over SP PPS in some warm season events when the freezing level was elevated. However, it had relatively large random errors due to its high sensitivity to errors in $Z_{\mathrm{DR}}$ (Cocks et al. 2017). DPR QPE is also subject to discontinuities and biases near the melting layer (Cocks et al. 2016).

Recent DP radar QPEs utilizing specific attenuation $A$ have shown less sensitivities to calibration errors in $Z$ and $Z_{\mathrm{DR}}$ (Ryzhkov et al. 2014; Wang et al. 2014, 2019; Cocks et al. 2019; Ryzhkov and Zrnić 2019), and a higher linearity was found between $R$ (rain rate) and $A$ than between $R$ and other radar variables (Ryzhkov et al. 2014; Ryzhkov and Zrnić 2019). Further, $A$ is immune to partial beam blockages (Cocks et al. 2019). While $K_{\mathrm{DP}}$ has similar advantages, the estimation of $K_{\mathrm{DP}}$ requires smoothing along the radial which results in a degradation of the spatial resolution. Conversely, $A$ provides rain rate estimates at the native radar resolution in a wider range of rain intensities as opposed to $K_{\mathrm{DP}}$, which is noisy in light rain (Ryzhkov et al. 2014).

Despite the successes, previous $R(A)$ studies were limited in single radar framework and only applied in areas where the radar beam is below the melting layer (ML). Further, those studies focused mainly on wide spread heavy rain near the radar. To make $R(A)$ useful in an operational environment across a large national radar network, two additional steps are necessary: 1$)$ to combine $R(A)$ with other radar 


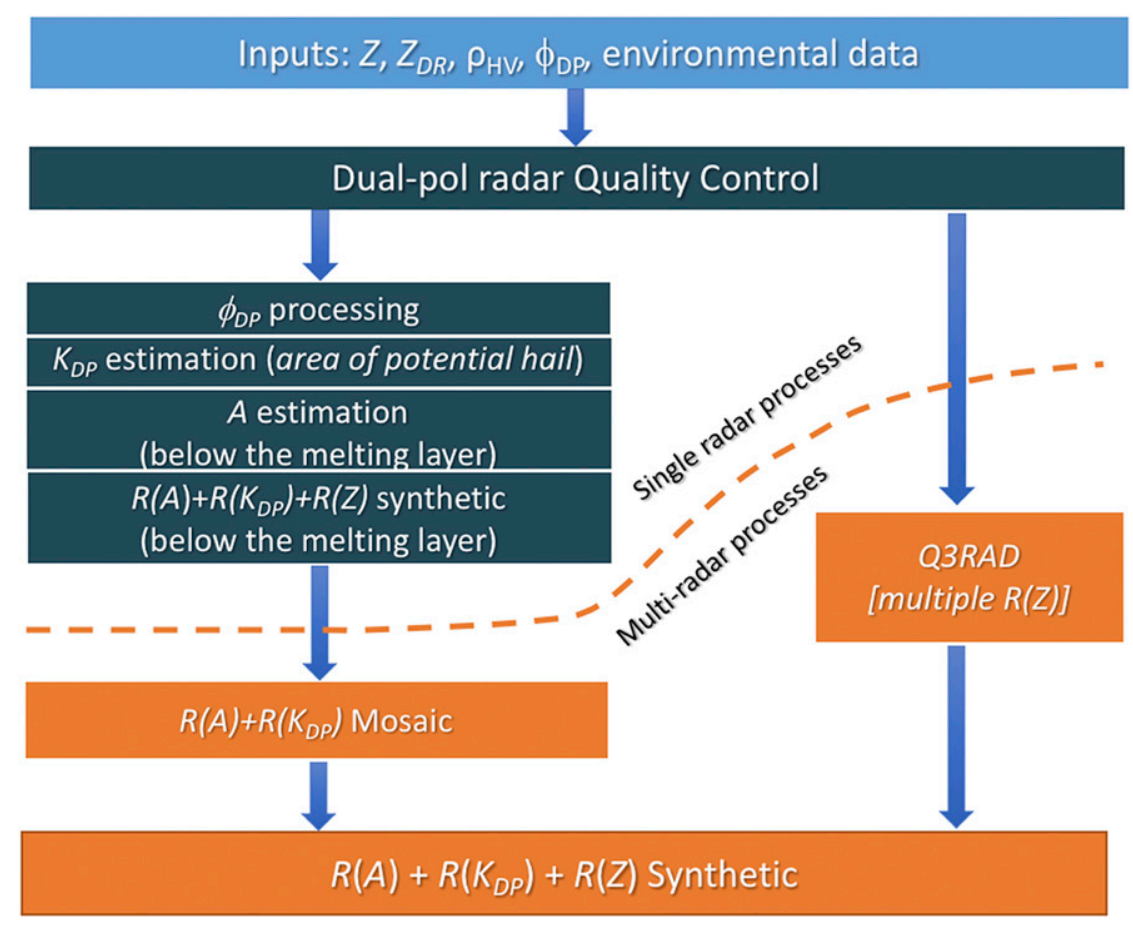

FIG. 1. An overview flowchart of the MRMS DP radar synthetic QPE.

QPE techniques and establish a synthetic scheme that is applicable everywhere and 2) to assess the $R(A)$ performance for all rain regimes including light and sporadic ones and to determine an optimal combination of $R(A)$ and other radar QPE relationships. The current study focused on these two steps and developed a seamless DP synthetic QPE for the conterminous United States (CONUS) domain within the Multi-Radar Multi-Sensor (MRMS; Zhang et al. 2016) system.

The MRMS DP synthetic QPE ("Q3DP" in short for the third generation of MRMS radar QPE) calculates $\mathrm{R}$ based on a combination of $A, K_{\mathrm{DP}}$, and $Z$. While $A$ has the aforementioned advantages for rain rate estimation, it is not applicable in radar observations that contain ice. Therefore, Q3DP applies $R(A)$ relationship in areas where radar is observing pure rain and $R\left(K_{\mathrm{DP}}\right)$ in areas with potential presence of hail. The area of pure rain was defined as where the radar observations were below the ML bottom and $Z<50 \mathrm{~dB} Z$. The ML bottom was determined from the temperature sounding at the radar site and the correlation coefficient $\rho_{\mathrm{HV}}$ field (Wang et al. 2019). The area of potential hail is defined as $Z \geq 50 \mathrm{~dB} Z$. The $R(Z)$ with a vertical profile of reflectivity (VPR) correction (Zhang and Qi 2010; Zhang et al. 2012a) was applied within and above the ML.

The Q3DP was tested, evaluated and refined in real-time across CONUS since October 2016. This paper provides an overview of the DP radar synthetic QPE and its performance during September and October 2018, where a relatively stable $R(A)$ version was applied. Section 2 describes the Q3DP methodology and section 3 presents the evaluation results across CONUS during September and October 2018. A summary follows in section 4 .

\section{Methodology}

Figure 1 shows an overview flowchart of the MRMS DP synthetic QPE process. The input data includes DP radar moments and environmental data such as the $3 \mathrm{D}$ temperature field on constant heights above the mean sea level, freezing level height and surface wet bulb temperature. A DP radar data quality control (QC; Tang et al. 2014) is applied to remove nonprecipitation echoes. After the QC, the differential phase $\phi_{\mathrm{DP}}$ field is further processed for additional quality assurance. The $\phi_{\mathrm{DP}}$ processing include four steps. The first step includes a speckle filter and a $\rho_{\mathrm{HV}}$ screening. The speckle filter checks for each $\phi_{\text {DP }}$ pixel and counts the number of nonmissing $\phi_{\text {DP }}$ pixels in a $4.5^{\circ} \mathrm{km} \times$ $2.25 \mathrm{~km}$ box centered at the given pixel. If the number is less than $50 \%$ of the box total, then the given $\phi_{\text {DP }}$ pixel at the center is removed. A $\rho_{\mathrm{HV}}>0.8$ filter was applied to remove noisy and unreliable $\phi_{\mathrm{DP}}$ data potentially associated with hail, severe nonuniform beam filling, or other residual contaminations after the DP QC. The second step unfolds the $\phi_{\mathrm{DP}}$ field based on the gate-to-gate $\phi_{\mathrm{DP}}$ change and adjusts the unfolded $\phi_{\mathrm{DP}}$ field to assure a monotonic increase trend with range. The third step applies a $6.25 \mathrm{~km}$ radial running mean through all nonmissing $\phi_{\mathrm{DP}}$ pixels to further reduce random errors and fluctuations. Finally, a linear interpolation along the radial is applied to fill in any gaps in the $\phi_{\mathrm{DP}}$ field.

Specific differential phase $K_{\mathrm{DP}}$ is calculated via a local linear fitting to $\phi_{\mathrm{DP}}$ along the radial direction in areas where hail might be present. Specific attenuation $A$ is calculated following the steps presented in Wang et al. (2019) and the steps are briefly reintroduced below for easy reference: 

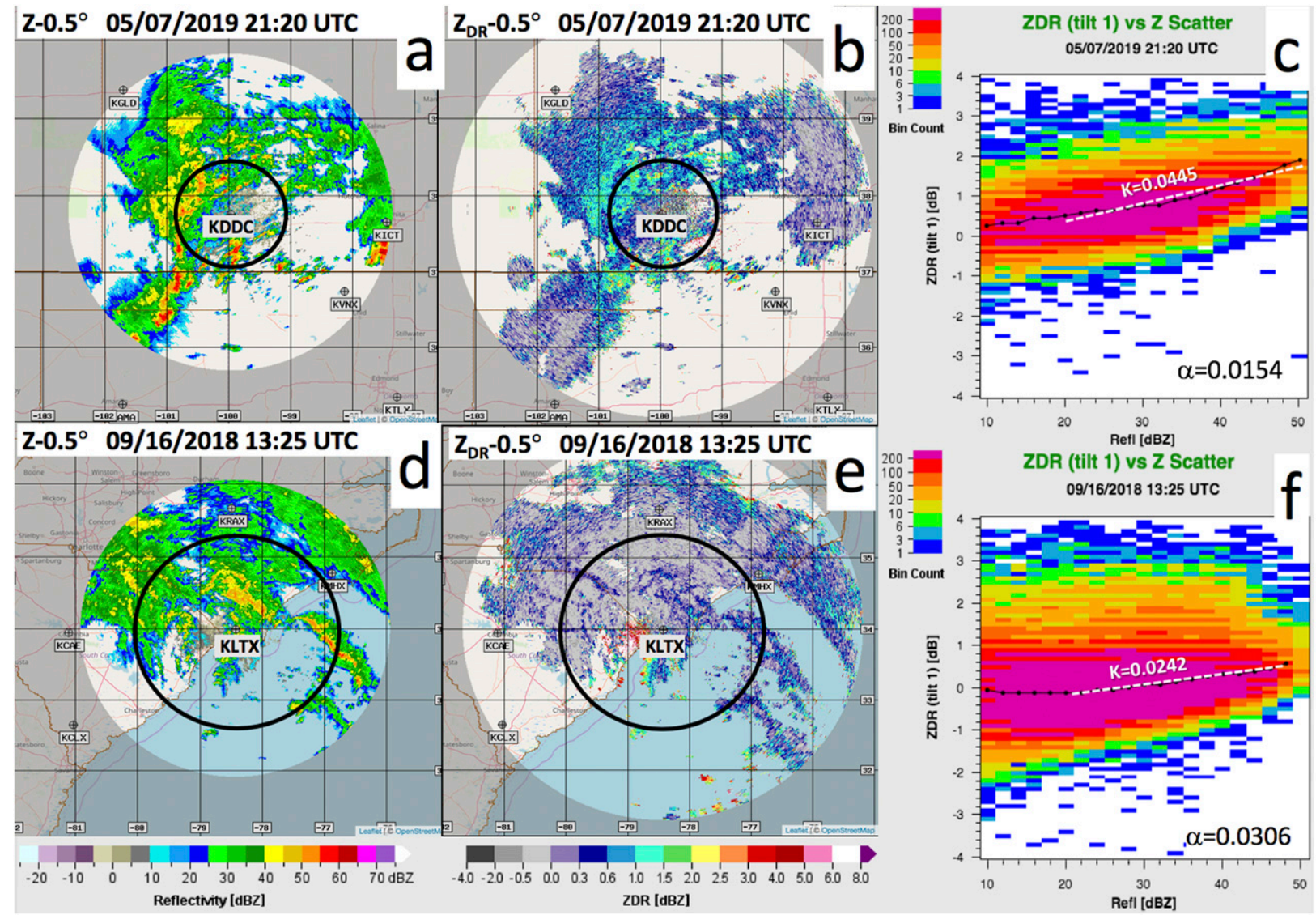

FIG. 2. (a),(d) Reflectivity $Z$; (b),(e) differential reflectivity $Z_{\mathrm{DR}}$; and (c),(f) $Z-Z_{\mathrm{DR}}$ scatterplot from (top) KDDC at 2120 UTC 7 May 2019 and (bottom) KLTX at 1325 UTC 16 Sep 2018. The black circles indicate the estimated bottom of the melting layer. The black dots in (e) and (f) indicate median $Z_{\mathrm{DR}}$ values, and the white dashed lines represent the linear fit to the median $Z_{\mathrm{DR}}$ vs $Z$.

$$
A(r)=\frac{Z_{a}^{b}(r) C(b, \text { PIA })}{I\left(r_{1}, r_{2}\right)+C(b, \text { PIA }) I\left(r, r_{2}\right)},
$$

where

$$
\begin{aligned}
I\left(r_{1}, r_{2}\right) & =0.46 b \int_{r_{1}}^{r_{2}} Z_{a}^{b}(s) d s \\
I\left(r, r_{2}\right) & =0.46 b \int_{r}^{r_{2}} Z_{a}^{b}(s) d s \\
C(b, \mathrm{PIA}) & =\exp (0.23 b \mathrm{PIA})-1, \\
\operatorname{PIA}\left(r_{1}, r_{2}\right) & =\alpha \Delta \phi_{\mathrm{Dp}} ; \quad \text { where } \quad \Delta \phi_{\mathrm{Dp}}=\phi_{\mathrm{Dp}}\left(r_{2}\right)-\phi_{\mathrm{Dp}}\left(r_{1}\right) .
\end{aligned}
$$

Here $r$ is the range at a given gate, $r_{1}$ is the nearest gate with precipitation in a given radial and $r_{2}$ the last precipitation gate or the gate just below the bottom of the melting layer, whichever is closer to the radar. The ML bottom was approximated by a weighted mean of the $10^{\circ}$ and $0^{\circ} \mathrm{C}$ heights at the radar site and refined by the correlation coefficient $\left(\rho_{\mathrm{HV}}\right)$ field (Wang et al. 2019). The $Z_{a}$ is the measured reflectivity, $b$ is a constant (0.62 for S band; Ryzhkov et al. 2014), PIA is the pathintegrated attenuation, and $\alpha$ is a parameter that generally varies with drop size distributions and with temperature (Ryzhkov et al. 2014; Wang et al. 2019). Pixels in $\left[r_{1}, r_{2}\right]$ with potential hail contamination are excluded from the PIA calculation. Note that reflectivity $Z_{a}$ in Eq. (1) appeared in both the nominator and denominator, which effectively removes any systematic biases in the reflectivity observations when calculating $A$. Therefore, $A$ is insensitive to systematic biases in $Z_{a}$ such as those from partial blockage, calibration, and attenuation.

In the current scheme, $\alpha$ is estimated from the $0.5^{\circ}$ tilt data using the " $Z_{\mathrm{DR}}$ slope" $K$ :

$$
\alpha=-0.75 K+0.04875 \text {, }
$$

where $K$ is a linear fit to the median $Z_{\mathrm{DR}}$ values of each $2-\mathrm{dB} Z$ reflectivity bin between 20 and $50 \mathrm{~dB} Z$ range (Fig. 2). Equation (6) was obtained from disdrometer data and supported by an analysis of $\sim 10$ events with wide spread moderate to heavy precipitation events from different geographical regimes. Figure 2 illustrates the process of $\alpha$ calculation. For the mesoscale convective system (MCS) near KDDC (Figs. 2a-c) on 7 May 2019, there was an apparent increase of $Z_{\mathrm{DR}}$ with increasing $Z$, indicating the presence of large rain drops in the convective system. The $Z_{\mathrm{DR}}$ slope was relatively large $\left(K=0.0445 \mathrm{~dB} \mathrm{~dB} Z^{-1}\right)$ 


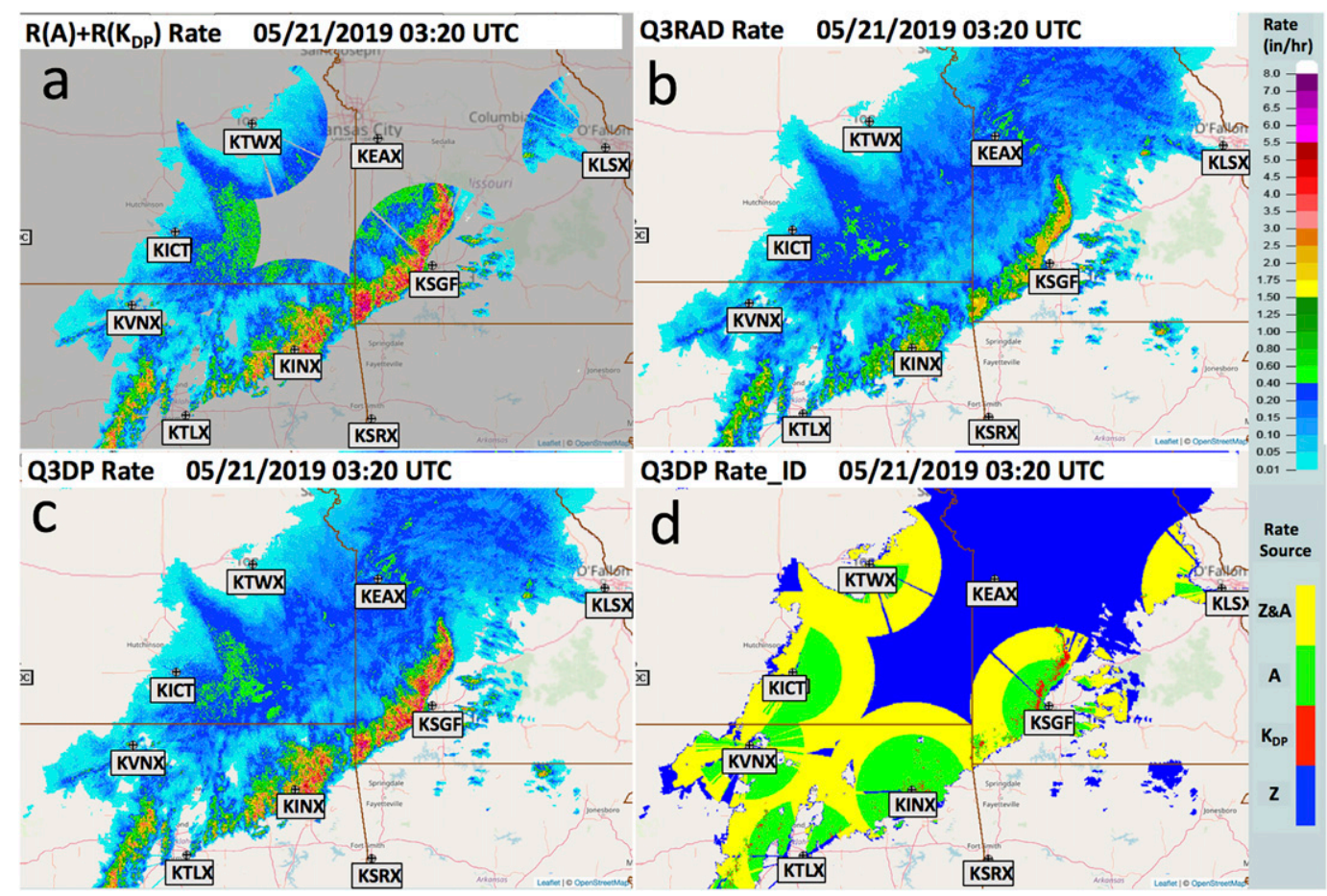

FIG. 3. Precipitation rate fields from the mosaic of (a) $R(A)+R\left(K_{\mathrm{DP}}\right)$, (b) multiple $R(Z)$, and (c) the final synthetic QPE. (d) A category field that represents the radar variables from which the rate was obtained. Green represents areas with $R(A)$, blue $R(Z)$, red $R\left(K_{\mathrm{DP}}\right)$, and yellow a weighted mean of $R(A)$ and $R(Z)$. The data were valid at 0320 UTC 21 May 2019.

and $\alpha$ relatively low $(0.0154)$ and close to those for convective rain (Ryzhkov et al. 2014; Cocks et al. 2019; Wang et al. 2019). For the Hurricane Florence case near KLTX (Wilmington, North Carolina), on 16 September 2018 (Figs. 2d-f), the $Z_{\mathrm{DR}}$ slope was relatively flat $(K=0.0242)$ and $\alpha(0.0306)$ was higher and closer to a tropical type rain value.

Equation (6) is used to estimate $\alpha$ only when there are sufficient $Z-Z_{\mathrm{DR}}$ data pairs in each $2-\mathrm{dB} Z$ bin between 20 and $50 \mathrm{~dB} Z$. If not enough $Z-Z_{\mathrm{DR}}$ data pairs are found between 20 and $50 \mathrm{~dB} Z$, the number of $Z-Z_{\mathrm{DR}}$ pairs in each $2-\mathrm{dB} Z$ bin between 10 and $30 \mathrm{~dB} Z$ is checked. If significant data samples were found between 10 and $30 \mathrm{dBZ}$, then the precipitation is considered pure stratiform and a default stratiform $\alpha(0.035)$ is applied. Otherwise, a new linear fit may be applied to the median $Z_{\mathrm{DR}}$ values between 10 and $40 \mathrm{~dB} Z$ range if sufficient $Z-Z_{\mathrm{DR}}$ pairs were found in that range. If insufficient data pairs were found between 10 and $40 \mathrm{dBZ}$, the precipitation is considered sporadic. A default convective (0.015) or stratiform (0.035) $\alpha$ is applied depending on the reflectivity intensities.

Once $\alpha$ is obtained and $A$ calculated for each radar gate, precipitation rates are estimated from $A$ on the $0.5^{\circ}$ tilt in areas where reflectivity is below $45 \mathrm{dBZ}$ (an adaptable parameter):
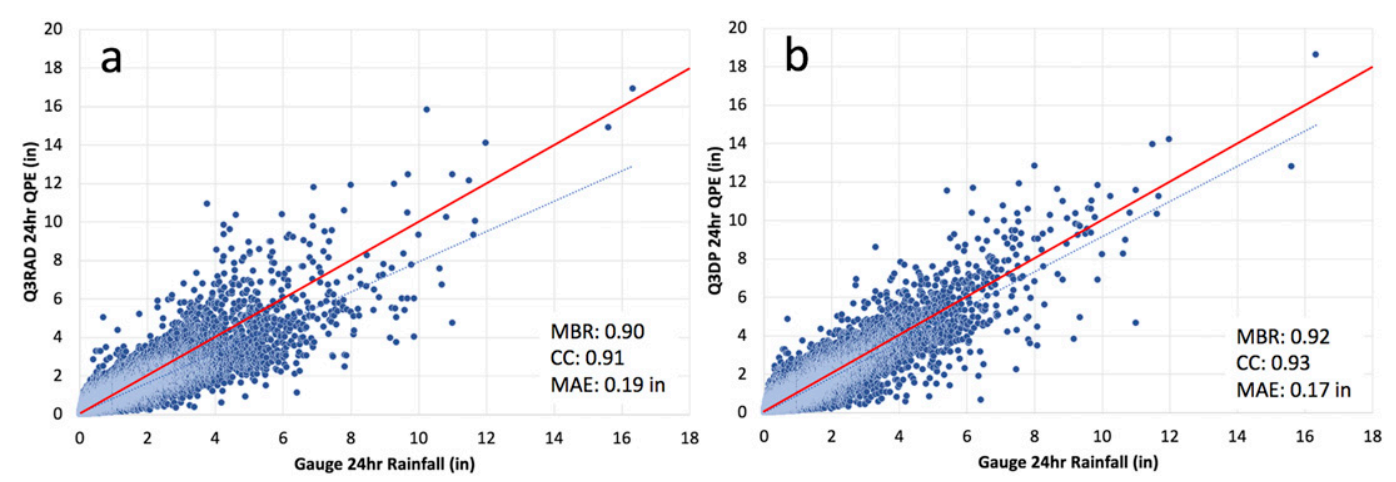

FIG. 4. (a) Q3RAD and (b) Q3DP 24-h QPE vs gauge daily observations for the period of 15 Sep-31 Oct 2018. Blue dotted lines show a linear fit to the data and red solid lines a 1:1 fit. 
TABLE 1. Hit/miss rates of the Q3RAD and Q3DP 24-h QPEs for predefined gauge 24-h rainfall categories. The bold numbers indicate the better hit rates among the two products.

\begin{tabular}{ccccccc}
\hline \hline Gauge 24h (in.) & & $G<0.5$ & $0.5 \leq G<1$ & $1 \leq G<2$ & $2 \leq G<4$ & $G \geq 4$ \\
\hline No. of samples & & 53478 & 13796 & 9834 & 3404 & H \\
Category & & VL & L & 0.03 & 0.00 & VH \\
Q3RAD 24h & VL & 0.93 & 0.31 & 0.39 & 0.04 & 0.49 \\
& L & 0.06 & 0.10 & 0.54 & 0.42 & 0.05 \\
Q3DP 24h & M & 0.01 & 0.00 & 0.04 & 0.00 & 0.49 \\
& H & 0 & 0.00 & 0.04 & 0.03 & 0.48 \\
& VH & 0 & 0.34 & 0.32 & 0.30 & 0.00 \\
& VL & $\mathbf{0 . 9 5}$ & 0.54 & 0.12 & 0.07 & 0.02 \\
& L & 0.05 & 0.00 & 0.00 & 0.06 & 0.29 \\
& M & 0.00 & 0.00 & & $\mathbf{0 . 6 9}$ \\
\hline
\end{tabular}

$$
R(A)=4120 A^{1.03}
$$

Here the $R(A)$ relationship is for $\mathrm{S}$ band at $20^{\circ} \mathrm{C}$ (Ryzhkov et al. 2014). The $45-\mathrm{dB} Z$ constraint is to prevent $R(A)$ from potential hail contamination. Above $50 \mathrm{dBZ}$ (an adaptable parameter), two $R\left(K_{\mathrm{DP}}\right)$ relationships are applied depending on the correlation coefficient $\rho_{\mathrm{HV}}$ field:

$$
\begin{aligned}
& R\left(K_{\mathrm{DP}}\right)=29.0\left|K_{\mathrm{DP}}\right|^{0.77}, \quad \text { if } \quad \rho_{\mathrm{HV}}<0.97, \\
& R\left(K_{\mathrm{DP}}\right)=44.0\left|K_{\mathrm{DP}}\right|^{0.822}, \text { if } \rho_{\mathrm{HV}} \geq 0.97 .
\end{aligned}
$$

Equation (8a) was derived using disdrometer measurements in the proximity of selected hailstorms in central Oklahoma, and Eq. (8b) provided optimal performance in heavy rain with large drops (Ryzhkov et al. 2005), also in central Oklahoma. Between 45 and $50 \mathrm{~dB} Z$, a linear combination of $R(A)$ and $R\left(K_{\mathrm{DP}}\right)$ is applied to create a smooth transition between the two rates. The $R(A)$ and $R\left(K_{\mathrm{DP}}\right)$ are calculated from the $0.5^{\circ}$ tilt currently with two additional constraints applied: 1) the beam blockage must be less than $90 \%$ and 2) $\Delta \phi_{\mathrm{DP}}$ must be greater than $0^{\circ}$. If the blockage is $\geq 90 \%$ or $\Delta \phi_{\mathrm{DP}} \leq 0^{\circ}$ in a given radial, the rate values for all the pixels in that radial are set to missing and $R(Z)$-based rates will be applied in the synthetic process at a later time (Fig. 1).

Precipitation rate fields estimated via $R(A)$ and $R\left(K_{\mathrm{DP}}\right)$ from individual radars are mosaicked through a physically based scheme shown in Qi and Zhang (2017). Since $R(A)$ is only valid below the melting layer, the mosaic has data voids in areas far away from the radars or when there are radar outages. Figure 3 a shows such a mosaic where KEAX radar was missing and the $R(A)+R\left(K_{\mathrm{DP}}\right)$ fields from the nearby radars (KTWX and KSGF) were not able to fill the gap. The $R(Z)$-based QPE (Q3RAD) (Fig. 3b) did not have this limitation and was able to capture the full area of precipitation. Q3RAD was based on an automated precipitation classification and multiple $R(Z)$ relationships and was described in Zhang et al. (2016). The multiple $R(Z)$ relationships include

$$
\begin{aligned}
\text { stratiform: } & R_{\text {stra }}=\max \left(0.0365 Z^{0.625}, 0.1155 Z^{0.5}\right) ; \\
\text { convective: } & R_{\text {conv }}=0.017 Z^{0.714} \\
\text { tropical: } & R_{\text {trop }}=\beta 0.010 Z^{0.833}
\end{aligned}
$$

where $\beta$ is a rate multiplier ranging from 1.0 to 1.5 depending on the month of the year and the proximity to frequent hurricane zones (Zhang et al. 2016; Cocks et al. 2017). For the coastal areas of the Gulf of Mexico, the Atlantic, and the southeastern United States, $\beta$ can be as high as 1.45 for September and 1.5 for October. The convective $R(Z)$ was capped at $49 \mathrm{~dB} Z$ $\left(53.8 \mathrm{~mm} \mathrm{~h}^{-1}\right.$ or $\left.2.1 \mathrm{in.} \mathrm{h}^{-1}\right)$ for hail.

Q3RAD is used to fill in precipitation areas in Q3DP where the radar beam intersects or overshoots the melting layer (Fig. 3c). To prevent discontinuities between $R(A)$ and $R(Z)$, a weighted mean of the two rates is applied in a transition zone (default width: $50 \mathrm{~km}$ ) near the outer boundary of $R(A)$ (i.e., the yellow colored area in Fig. 3d). Finally, an evaporation correction is applied to the mosaicked precipitation rate field based on the methodology given by Martinaitis et al. (2018). The correction factor is a function of the rate, the height of the radar estimate, and atmospheric properties between the radar data height and the ground. The correction helps reduce false light precipitation in the radar QPE due to virga.

\section{Evaluations}

Q3DP was evaluated across CONUS during the period of 15 September-31 October 2018. Figure 4 shows scatterplots of the Q3DP and Q3RAD 24-h QPEs versus CoCoRaHS (Community Collaborative Rain, Hail and Snow Network,

TABLE 2. Mean bias ratio (MBR), correlation coefficient (CC), mean absolute error (MAE), and fractional MAE of the Q3RAD and Q3DP 24-h QPEs for predefined gauge 24-h rainfall categories. The bold numbers indicate the better scores among the two products.

\begin{tabular}{rlrrrrr}
\hline \hline \multicolumn{2}{c}{ Category } & \multicolumn{1}{c}{ VL } & \multicolumn{1}{c}{ L } & \multicolumn{1}{c}{ M } & \multicolumn{1}{c}{ H } & \multicolumn{1}{c}{ VH } \\
\hline \multicolumn{2}{c}{ G-mean (in.) } & 0.17 & 0.71 & 1.37 & 2.66 & 5.45 \\
Q3RAD & MBR & 1.19 & 0.91 & 0.82 & 0.80 & 0.81 \\
& CC & $\mathbf{0 . 6 9}$ & 0.39 & 0.43 & 0.52 & 0.57 \\
Q3DP & MAE (in.) & 0.09 & $\mathbf{0 . 2 1}$ & 0.38 & 0.78 & 1.73 \\
& fMAE (\%) & 52.94 & $\mathbf{2 9 . 5 8}$ & 27.74 & 29.32 & 31.74 \\
& MBR & $\mathbf{1 . 0 4}$ & 0.91 & $\mathbf{0 . 8 8}$ & $\mathbf{0 . 9 2}$ & $\mathbf{0 . 9 5}$ \\
& CC & 0.68 & 0.39 & $\mathbf{0 . 4 9}$ & $\mathbf{0 . 5 9}$ & $\mathbf{0 . 7 1}$ \\
& MAE (in.) & $\mathbf{0 . 0 8}$ & 0.22 & $\mathbf{0 . 3 6}$ & $\mathbf{0 . 5 9}$ & $\mathbf{1 . 1 4}$ \\
& fMAE (\%) & $\mathbf{4 7 . 0 6}$ & 30.99 & $\mathbf{2 6 . 2 8}$ & $\mathbf{2 2 . 1 8}$ & $\mathbf{2 0 . 9 2}$ \\
\hline
\end{tabular}




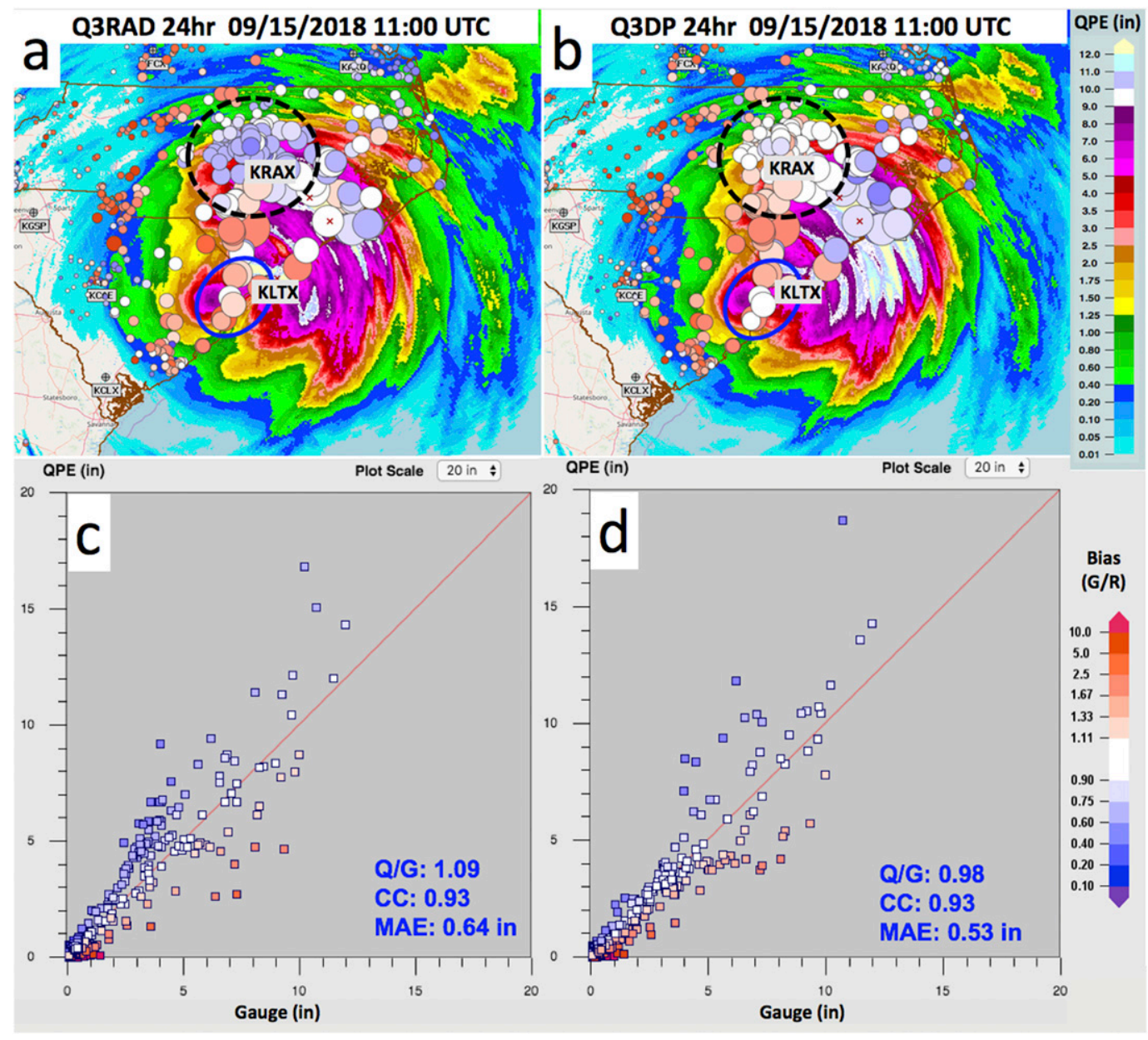

FIG. 5. The 24-h (a) Q3RAD and(b) Q3DP ending at 1100 UTC 15 Sep 2018 overlaid with dots representing CoCoRaHS gauge sites. The size of the dots represents gauge observed amounts and the color represents the gauge/QPE bias ratios. (c),(d) Scatterplots of the two QPEs in (a) and (b), respectively, vs gauges. The statistic scores in the scatterplots are the domain mean QPE/gauge bias ratio $(Q / G)$, correlation coefficient (CC), and mean absolute error (MAE). The solid blue and dashed black circles highlight areas where the Q3RAD and Q3DP had notable differences.

www.cocorahs.org/; Cifelli et al. 2005) gauge observations. The scores listed in the plots are defined as follows:

where

$$
\text { mean bias ratio }(\mathrm{MBR}): \mathrm{MBR}=\bar{Q} / \bar{G},
$$

$$
\begin{array}{r}
\bar{Q}=\frac{\sum_{i=1}^{N} Q_{i}}{N}, \\
\bar{G}=\frac{\sum_{i=1}^{N} G_{i}}{N},
\end{array}
$$

correlation coefficient (CC): CC $=\frac{\sum_{i=1}^{N}\left(Q_{i}-\bar{Q}\right)\left(G_{i}-\bar{G}\right)}{\sqrt{\sum_{i=1}^{N}\left(Q_{i}-\bar{Q}\right)^{2} \sum_{i=1}^{N}\left(G_{i}-\bar{G}\right)^{2}}}$, mean absolute error (MAE): MAE $=\frac{1}{N} \sum_{i=1}^{N}\left|Q_{i}-G_{i}\right|$,

$$
\text { fractional MAE: } \mathrm{fMAE}=100 \times \mathrm{MAE} / \bar{G} .
$$

The variable $N$ is the total number of QPE-gauge pairs for the given dataset; $Q_{i}$ and $G_{i}$ are the radar estimated and gauge observed 24-h rainfalls at the $i$ th gauge station, respectively.

Q3DP showed a reduction in systematic bias and random errors over Q3RAD, especially for the heavy amounts higher than 2 in. (Fig. 4). The improvements for the heavy amounts are also reflected in the hit/miss rates (Table 1) and the statistic scores (Table 2) for different rainfall categories. The categories are subjectively defined as very light (VL: $G<0.5 \mathrm{in}$.), light (L: $0.5 \leq G<1$ in.), moderate (M: $1 \leq G<2$ in.), heavy (H: $2 \leq G<4$ in.), and very heavy (VH: $G \geq 4$ in.). The hit rates of Q3DP were about the same as Q3RAD in the VL, L, and $\mathrm{M}$ categories (Table 1$)$ but significantly higher in the $\mathrm{H}(0.6$ vs 0.42$)$ and $\mathrm{VH}(0.69$ vs 0.48$)$ categories. Q3RAD had a $19 \%$ overestimation bias (Table 2) in the VL rain and 18\%, 20\%, 


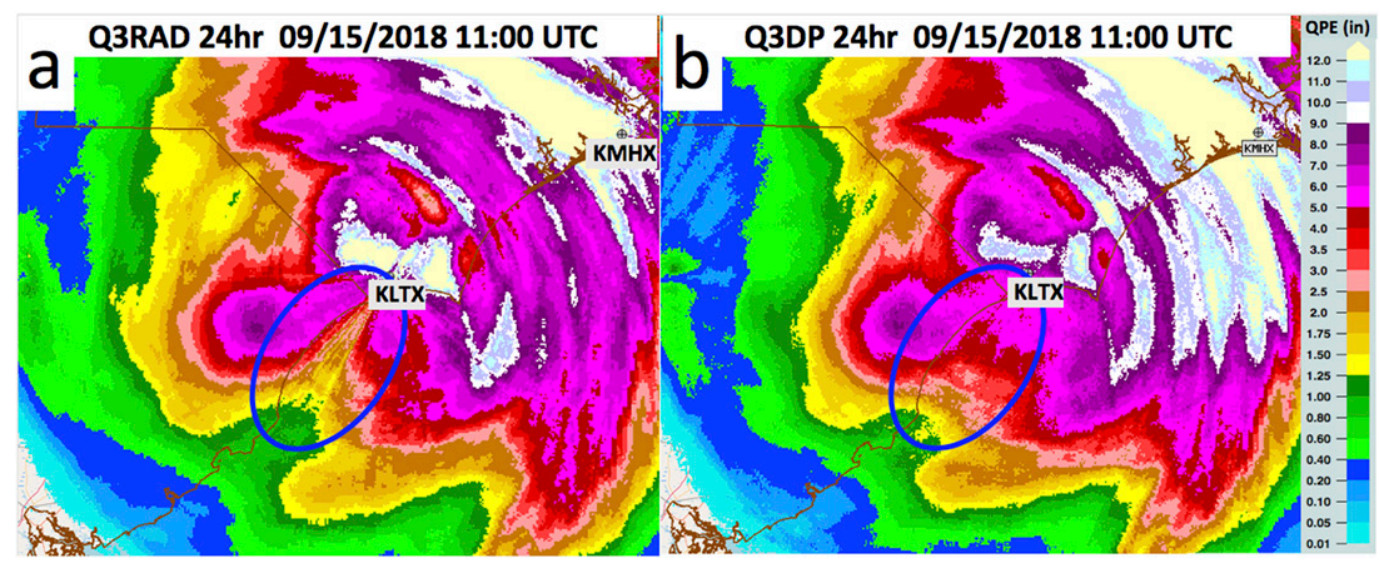

FIG. 6. The same (a) Q3RAD and (b) Q3DP as in Fig. 5, but zoomed into KLTX area and without the gauge overlay.

and $19 \%$ underestimation biases in the $\mathrm{M}, \mathrm{H}$, and $\mathrm{VH}$ rain, respectively. Q3DP reduced the biases by $15 \%, 6 \%, 12 \%$, and $16 \%$ for $\mathrm{VL}, \mathrm{M}, \mathrm{H}$, and $\mathrm{VH}$ categories, respectively, and remained the same for the L category. A similar trend was found in the MAE score, where Q3DP reduced the errors for $\mathrm{VL}, \mathrm{M}$, $\mathrm{H}$, and $\mathrm{VH}$ categories but slightly increased the error in the $\mathrm{L}$ category (Table 2). For correlation coefficient, Q3DP was about the same as Q3RAD in the VL and $\mathrm{L}$ and was higher in the $\mathrm{M}-\mathrm{VH}$ categories.

Q3DP showed a significantly reduced uncertainty with respect to Q3RAD for rainfall amounts above $\sim 3$ in. (Fig. 4). The reduced uncertainty in Q3DP was also reflected in its higher hit rates (Table 1) and lower MAEs/fMAEs (Table 2) for the $\mathrm{H}$ and $\mathrm{VH}$ categories. Q3DP reduced the fMAE by $\sim 6 \%, 7 \%$, and $11 \%$ for the $\mathrm{VL}, \mathrm{H}$, and $\mathrm{VH}$ categories, respectively, while the fMAEs of Q3DP and Q3RAD for the L and $M$ categories remained similar. Q3DP appeared to be slightly inferior to Q3RAD in the light rain category (Tables 1 and 2). Detailed analyses of individual events are presented below to help understand the potential physical processes associated with the aforementioned error characteristics.

Figure 5 shows the Hurricane Florence event in the first $24 \mathrm{~h}$ after its landfall. Q3RAD and Q3DP had similar MBRs (1.09 vs 0.98$)$ and CCs (0.93 vs 0.93$)$, while the latter had a lower MAE (0.64 vs 0.53 in., Fig. 5). Q3RAD was running an enhanced tropical $R-Z$ [ $\beta=1.45$, Eq. (11)] for most of the region throughout the period yet still underestimated around KLTX when compared to CoCoRaHS gauges (blue circle, Fig. 5a). Q3DP had less underestimation and matched gauge observations better (blue circle, Fig. 5b) than did the Q3RAD estimates. The underestimation in Q3RAD was likely due to a tree blockage to the southwest of KLTX (Raleigh-Durham, North Carolina; blue circle, Fig. 6a). The $R(A)$-based QPE was less impacted by the blockage (blue circle, Fig. 6b) and yielded QPE amounts that matched better with gauges (Fig. 5b).

Q3RAD had an overestimation around KRAX (black dashed circle; Fig. 5a), which was likely due to dry air entrainments to the northwest of the hurricane and a lower precipitation efficiency than in coastal areas. The enhanced tropical $R(Z)$ was not representative of the liquid water content in this region. Time series of the hourly MBR (Fig. 7) from Q3RAD, Q3DP, and Q3DP without the evaporation correction within $\sim 80 \mathrm{~km}$ of KRAX showed that the $R(Z)$-based estimates were on average $\sim 50 \%$ higher than the gauge observed values. The $R(A)$-based estimates reduced the wet bias to $\sim 13 \%$ (Fig. 7 ), demonstrating the advantage of $R(A)$ over $R(Z)$ in accurately capturing the liquid water amount. The evaporation had little effect in this case due to the heavy rain and moist environment.

Figure 8 shows an event of fast moving MCSs in South Dakota and Minnesota on 18 September 2018 that was associated with strong winds and hail. Q3RAD (Fig. 8a) was primarily using the convective $R-Z$ relationship [Eq. (9)] with a cap of $53.8 \mathrm{~mm} \mathrm{~h}^{-1}$ in hail areas (Zhang et al. 2016). It had a $54 \%$ overestimation bias on the 24-h scale (Fig. 8d) and the overestimation was especially high near KMPX (Minneapolis, Minnesota) radar (white circle, Fig. 8a). Based on storms reports of strong winds up to $60 \mathrm{kt}\left(\sim 31 \mathrm{~m} \mathrm{~s}^{-1}\right)$ during the event (https://www.spc.noaa.gov/climo/reports/180917_rpts.html), there were likely issues of gauge undercatch and/or clogging by hailstones. As a result, the actual overestimation may not be as high as $50 \%$. The $R(A)+R\left(K_{\mathrm{DP}}\right)$ reduced the overestimation by $8 \%$ (Fig. $8 \mathrm{e}$ vs Fig. $8 \mathrm{~d}$ ) and the evaporation correction further reduced it by $12 \%$ (Fig. $8 \mathrm{f}$ vs Fig. $8 \mathrm{e}$ ). The evaporation correction was found to be a main contributor to the improved hit rate of Q3DP over Q3RAD (Table 1) and reduced bias (Table 2) in the VL category.

Figure 9 shows a large-scale heavy rain event associated with two slow moving frontal systems across northern Oklahoma and central Texas. Q3RAD was mostly based on an enhanced tropical $R(Z)[\beta \approx 1.2$, Eq. (11)] during the event and had a systematic underestimation bias for rainfall amounts greater than 2 in. (Fig. 9c). Q3DP reduced the overall bias by $17 \%$ (Fig. 9d) and the MAE was reduced by $\sim 22 \%$ (Fig. 9d vs Fig. 9c). For rainfall amounts greater than 4 in., Q3DP reduced the bias by $35 \%$ and MAE by $44 \%$. The improvements were mostly in areas close to the radars [KFWS (Ft. Worth, TX), KFDR (Frederick/Altus Air Force Base, OK), KINX (Tulsa, 


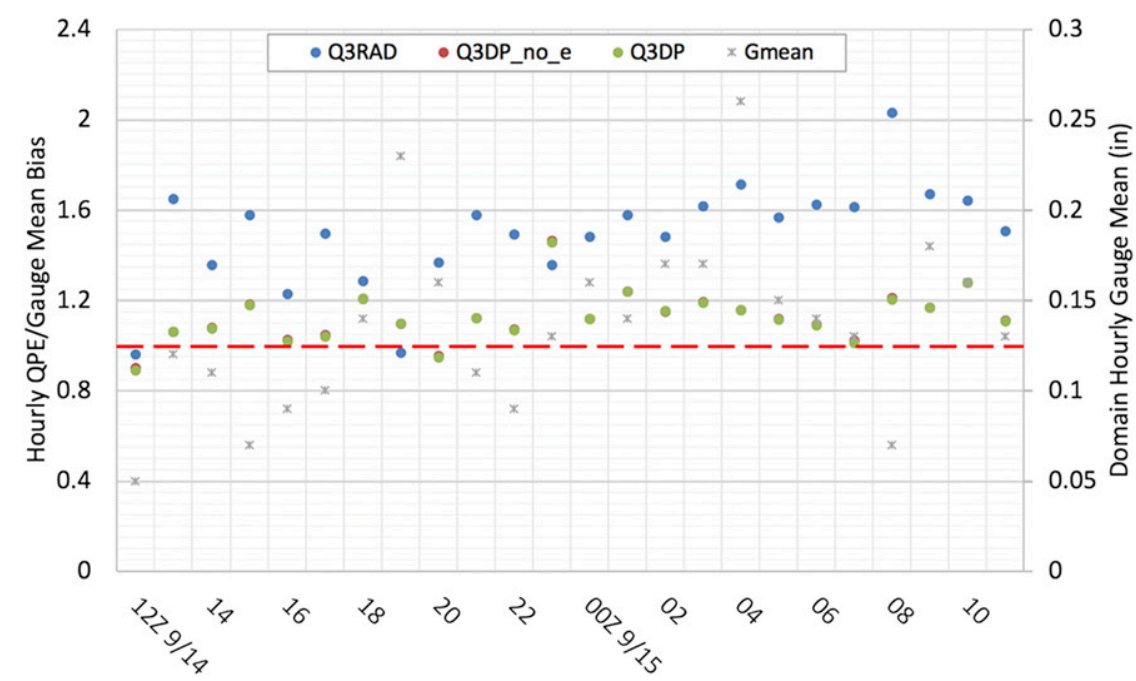

FIG. 7. Time series of hourly mean bias ratios for Q3RAD (blue dots), Q3DP (green dots), and Q3DP without evaporation correction (red dots) from 1200 UTC 14 Sep to 1100 UTC 15 Sep 2018. The red dashed line indicates a bias ratio of 1.00 (i.e., no bias). The domain average hourly gauge observations are shown as gray stars.

OK), KSRX (Fort Smith, AR), KLZK (Little Rock, AR)] and attributed to $R(A)$. This and the Hurricane Florence event demonstrated the superior performance of $R(A)$ in heavy to very heavy rainfalls and are consistent with the better hit rates and lower biases and MAEs in Q3DP (Tables 1 and 2).
Figure 10 shows a synoptic-scale precipitation system with a mix of convective and stratiform precipitation in the northeastern United States. Q3RAD was running convective $R(Z)$ [Eq. (10)] in the leading edge and stratiform $R(Z)$ [Eq. (9)] in the trailing region over $90 \%$ of the time. The enhanced tropical

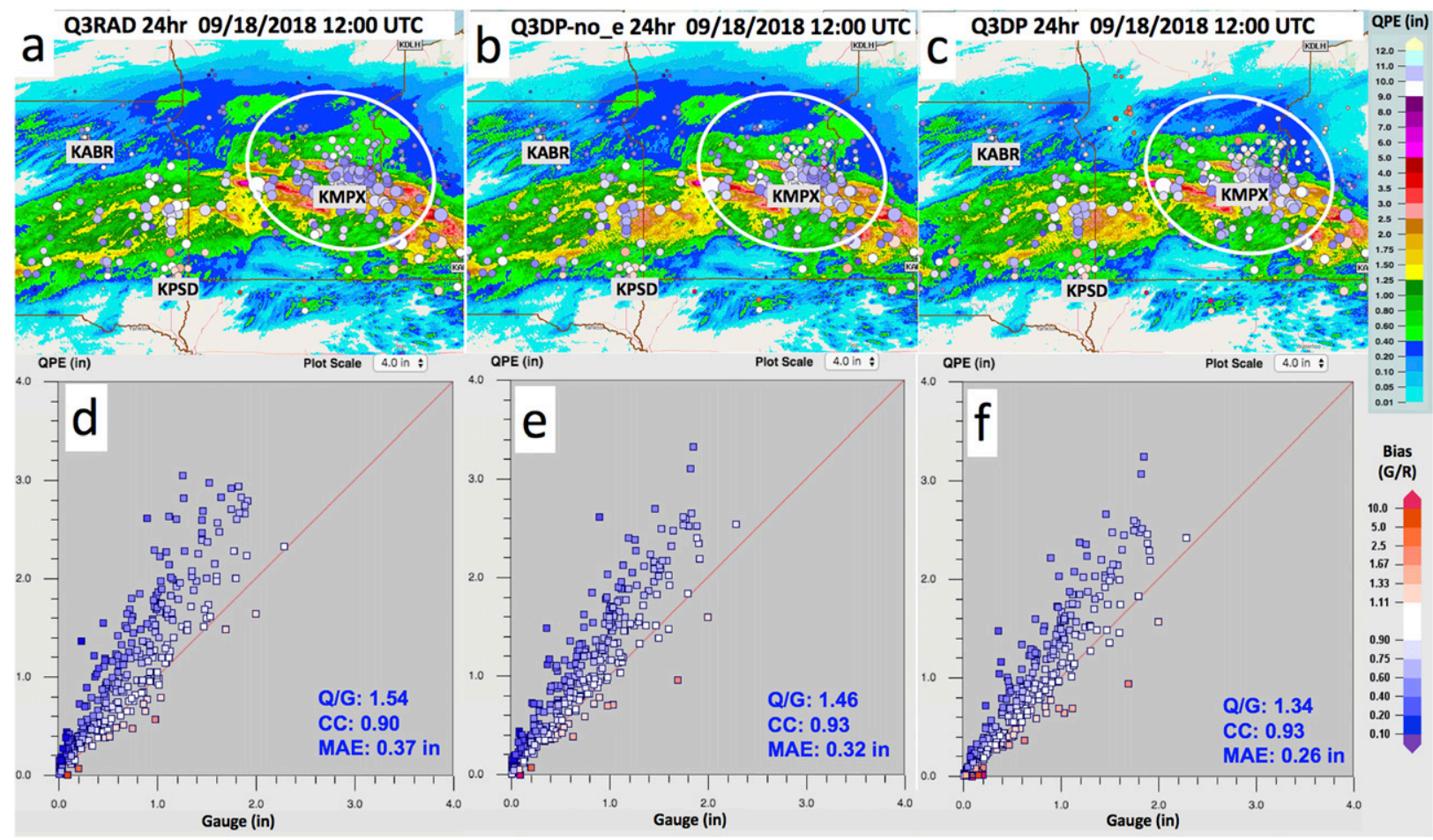

FIG. 8. As in Fig. 5, but for the 24-h (a),(d) Q3RAD; (b),(e) Q3DP without the evaporation correction; and (c),(f) Q3DP ending at 1200 UTC 18 Sep 2018 in South Dakota and Minnesota. The white circle indicates an area of significant overestimation in Q3RAD. 


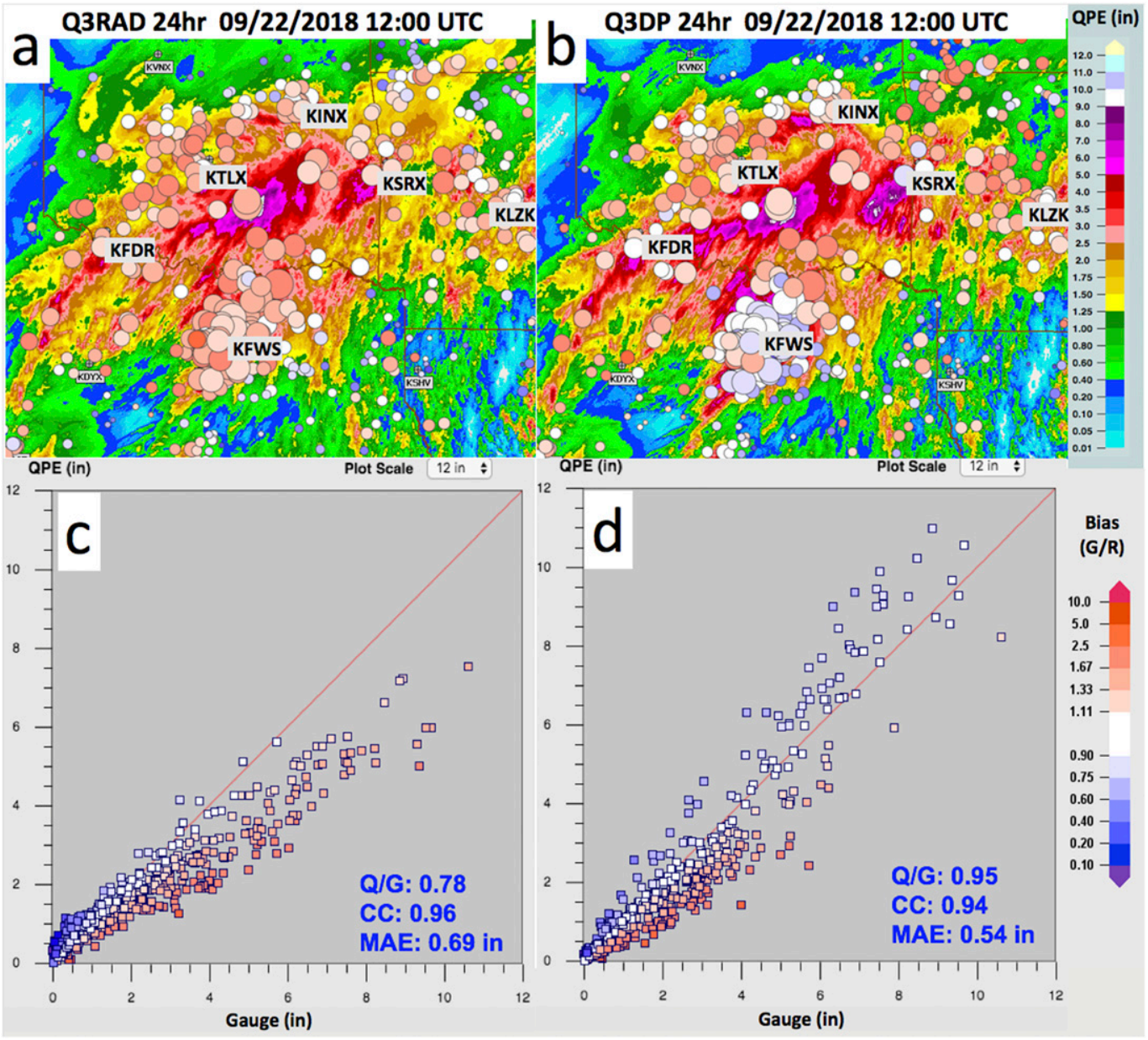

FIG. 9. As in Fig. 5, but for 24-h (a),(c) Q3RAD and (b),(d) Q3DP ending 1200 UTC 22 Sep 2018 in Oklahoma, northern Texas, and western Arkansas.

$R(Z)[\beta \approx 1.5$, Eq. (11)] was applied in small areas around KBOX (Boston, Massachusetts) during 0600-0800 UTC 3 October 2018. Q3RAD performed well when compared with the gauges in the southern half of the domain but had significant underestimation in the northern half where precipitation was mainly stratiform (Figs. 10a,c).

Q3DP reduced the underestimation bias in Q3RAD, especially for rainfall amounts greater than 2 in. (red dashed line, Fig. 10d vs Fig. 10c). However, Q3DP had a significant overestimation around KBOX (black circle in Fig. 10b and blue dashed line in Fig. 10d). The $Z$ and $Z_{\mathrm{DR}}$ fields (Figs. 11a,b) from 0535 UTC 3 October 2018 indicated moderate convective rain in the leading edge with $Z=45-50 \mathrm{~dB} Z$ (Fig. 11a) and $Z_{\mathrm{DR}}=0.6-1.5 \mathrm{~dB}$ (Fig. 11b). Due to the relatively low intensities of the convection, there was not enough $Z-Z_{\mathrm{DR}}$ samples above $44 \mathrm{~dB} Z$ (Fig. 11c) for a "valid" $Z_{\mathrm{DR}}$ slope between 20 and $50 \mathrm{~dB} Z$ range. As a result, $R(A)$ scheme applied the default stratiform $\alpha(0.035)$ for the entire precipitation period. A relationship between $\alpha$ and $Z_{\mathrm{DR}}$ from a large disdrometer dataset for S band (Fig. 2 in Wang et al. 2019) indicated that $\alpha$ varied between 0.024 and 0.014 for $Z_{\mathrm{DR}}$ of $0.6-1.5 \mathrm{~dB}$. The default stratiform $\alpha$ might have been too high and contributed to the overestimation in Q3DP around KBOX. A modified $R(A)$ scheme that adjusts the rate based on local $Z$ and $Z_{\mathrm{DR}}$ distributions is currently undergoing tests and will likely mitigate such overestimation errors with mixed stratiform and weak convective rain regimes.

The Q3DP and Q3RAD performance around KGYX (Portland, Maine) was mixed. Q3RAD had apparent blockage artifacts to the southeast of KGYX (white circle, Fig. 10a), which was known to be tree-related. The $R(A)$ mitigated the blockage artifacts and resulted in slightly higher amounts in the area (white circle, Fig. 10b) but still had an underestimation compared to gauges. To the north where the rain was lighter, $R(A)$ QPE had lower amounts and more underestimation than Q3RAD. The underestimation was likely due to two factors: $1)$ the differential phase changes $\left[\Delta \phi_{\mathrm{DP}}\right.$; Eq. (5)] in the radials were too small; and 2) the default stratiform $\alpha(0.035)$ was too low and unrepresentative of drop size distributions (DSDs) in the region. The current Q3DP applies $R(A)$ in all radials with $\Delta \phi_{\mathrm{DP}}>0^{\circ}$ (section 2). However, attenuation may be too weak to provide a useful $R(A)$ estimate when $\Delta \phi_{\text {DP }}$ was $<3^{\circ}$. 


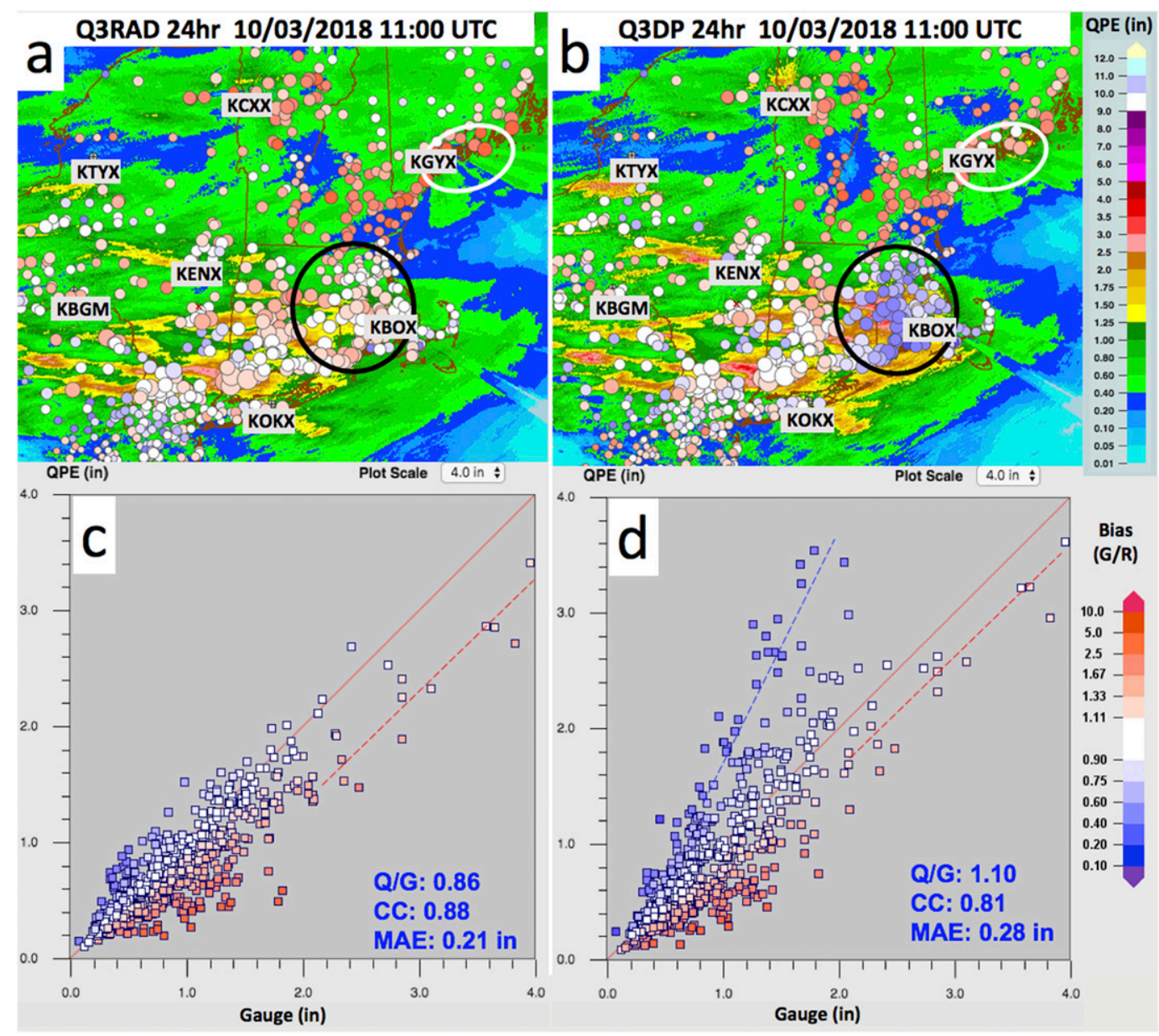

FIG. 10. As in Fig. 5, but for 24-h (a),(c) Q3RAD and (b),(d) Q3DP ending at 1100 UTC 3 Oct 2018 in the northeast. The black circle indicates an area of significant Q3DP overestimation and white circle an area of tree blockage. The blue (red) dashed lines in (c) and (d) indicate different overestimation (underestimation) of Q3RAD and Q3DP with respect to gauges.

A refined $R(A)$ scheme with higher $\Delta \phi_{\mathrm{DP}}$ thresholds $\left(2^{\circ}-3^{\circ}\right)$ is currently undergoing tests to mitigate such underestimation. When $\Delta \phi_{\mathrm{DP}}$ is below the predefined threshold, $R(Z)$ will be used instead of $R(A)$.

The reflectivity to the north of KGYX was mostly below $30 \mathrm{~dB} Z$ (Fig. 11d) and $Z_{\mathrm{DR}}$ was below $0.3 \mathrm{~dB}$ (Fig. 11e) during the event. The $\alpha-Z_{\mathrm{DR}}$ relationship in Wang et al. (2019) indicated that for $\mathrm{S}$ band at $\sim 20^{\circ} \mathrm{C}, \alpha$ value was $0.04-0.08$ for $Z_{\mathrm{DR}}$ values of $0.3 \mathrm{~dB}$ or less, which was much higher than the default stratiform $\alpha$ of 0.035 (Fig. 11f). The low $\alpha$ used in this case was likely a contributing factor of $R(A)$ underestimation in the low $Z /$ low $Z_{\mathrm{DR}}$ area to the north of KGYX as well as to the south. This is an opposite problem to the overestimation near $\mathrm{KBOX}$, but both situations indicated a need to refine the default stratiform $\alpha$ for an improved $R(A)$ accuracy in areas of light stratiform rain (underestimation) and in areas of mixed stratiform/weak convective rain (overestimation).

Figure 12 shows an event of mixed stratiform and strong convective rain in Nebraska, Kansas and Oklahoma where Q3DP had an underestimation. Q3RAD was applying convective $R(Z)$ in the leading edge and stratiform $R(Z)$ in the trailing stratiform region during the event. Q3RAD performed well when compared with the gauges except for an area midway between KUEX (Hastings, Nebraska) and KICT (Wichita, Kansas) where some overestimation was observed (black dashed line, Fig. 12a). An examination of reflectivity and correlation coefficient fields (not shown) indicated that the overestimation was a result of bright band contamination. While a VPR correction (Zhang and Qi 2010; Zhang et al. 2016) was applied in Q3RAD to mitigate such contamination, the correction assumed an isotopic bright band structure in the radar domain and was not as effective when the bright band was highly nonisotropic, as it was in this case. An enhanced VPR correction scheme based on dual-polarization variables and azimuthally varying bright band structure is under development in Q3DP and will further reduce the bright band contamination.

Q3DP had distinct underestimation errors around KUEX and KICT (black circles, Fig. 12b). The underestimation around KUEX was associated with light stratiform rain and unrepresentative default $\alpha(0.035)$ for low $Z /$ low $Z_{\mathrm{DR}}$ areas and 

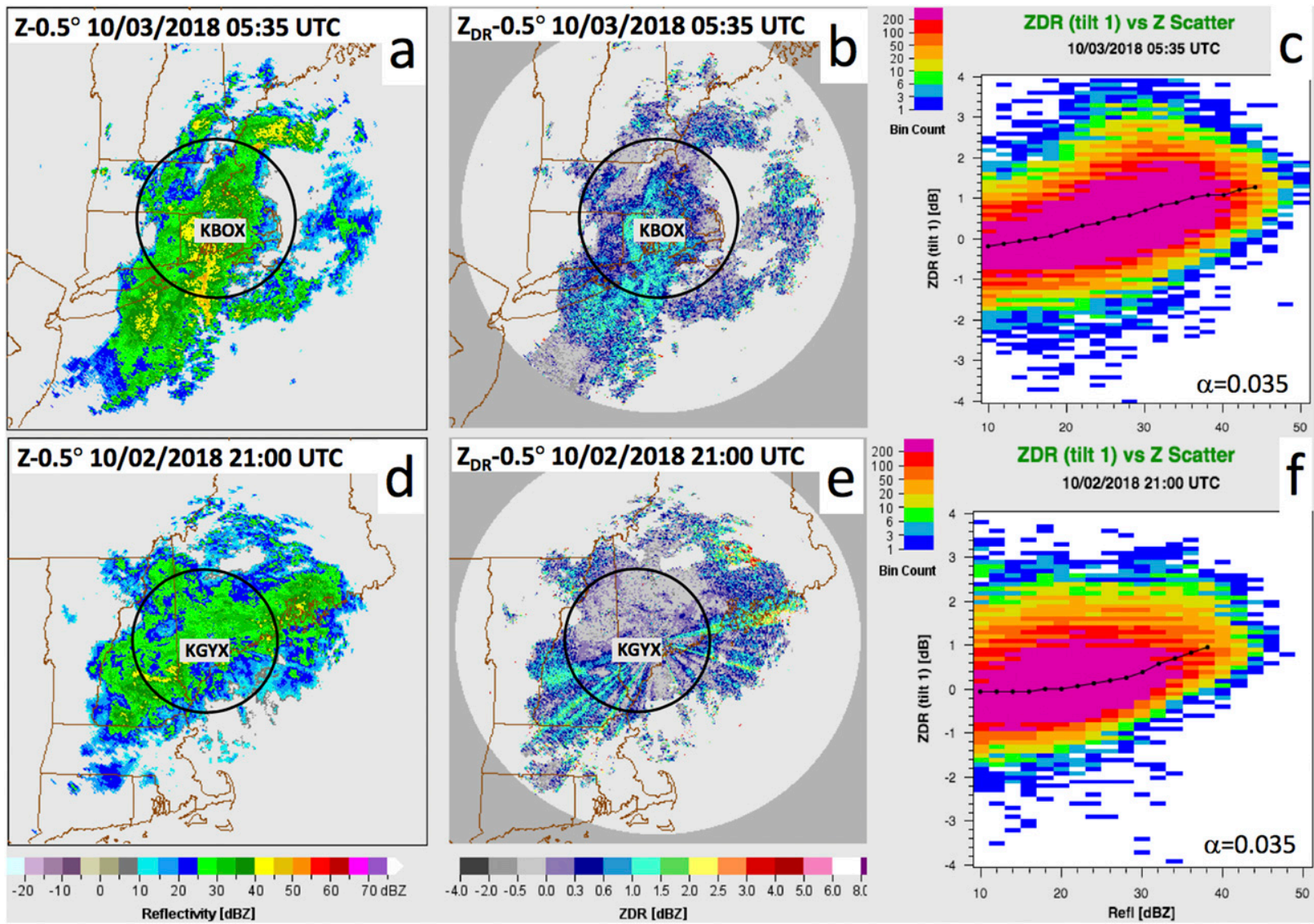

FIG. 11. (a),(d) Reflectivity $Z$; (b),(e) differential reflectivity $Z_{\mathrm{DR}}$; and (c),(f) $Z-Z_{\mathrm{DR}}$ scatterplot from the $0.5^{\circ}$ tilt of (top) KBOX at 0535 UTC 3 Oct and (bottom) KGYX at 2100 UTC 2 Oct 2018. The black circles indicate the estimated bottom of the melting layer.

was similar to the 3 October KGYX case. An examination of hourly Q3DP QPEs near KICT revealed that the most significant underestimation errors occurred between 0200 and 0600 UTC 9 October 2018, where a stratiform rain was present to the northwest of the radar and a strong convective rain was to the southeast (Figs. 13a,b). The strong convective rain dominated the $Z_{\mathrm{DR}}$ slope (Fig. 13c) and resulted in $\alpha$ values ranging from 0.018 to 0.020 during $0200-0600$ UTC. These $\alpha$ values are more representative of the convective precipitation DSDs to the southeast but not for the stratiform rain to the northwest where $\alpha$ should be higher $(>0.025)$ based on the low $Z(<35 \mathrm{~dB} Z)$ and $Z_{\mathrm{DR}}(<0.5 \mathrm{~dB})$ values. The unrepresentative $\alpha$ resulted in the underestimation in Q3DP (black circle at KICT, Fig. 12b vs Fig. 12c). Such underestimation errors in mixed stratiform/strong convective rain and overestimation in mixed stratiform/weak convective rain (e.g., KBOX event on 3 October) had contributed to the uncertainties in Q3DP for daily rainfalls below 3 in. Meanwhile, the underestimation errors in light stratiform rain (e.g., KGYX on 3 October and KUEX on 9 October) associated with the default stratiform $\alpha$ (0.035) had contributed to the lower hit rate (Table 1) and larger MAE in Q3DP for the L category (Table 2) than in Q3RAD. The local adjustment to the $R(A)$ scheme based on $Z$ and $Z_{\mathrm{DR}}$ distributions as mentioned earlier should mitigate these errors and improve the Q3DP performance in light rain category.

Figure 14 shows a heavy rain event associated with Hurricane Michael after its landfall. Q3DP had less underestimation bias ( $10 \%$ vs $17 \%)$, higher correlation coefficient (0.9 vs 0.87 ) and lower MAE ( 0.40 vs 0.48 in.) than Q3RAD. One notable difference between Q3DP and Q3RAD was with the heavy rainfall between KGSP and KMRX (black circle, Figs. 14a,b) where Q3RAD had significant underestimation and Q3DP compared well with gauge observations. A typical high-efficiency $\alpha$ ranging from 0.0287 to 0.0390 was applied in $R(A)$ and contributed to a more accurate estimation (black circle, Fig. 14b). In Q3RAD, a mixture of stratiform [Eq. (9)] and enhanced tropical $[\beta=1.5$; Eq. (11)] $R(Z)$ were applied throughout the event in the area. The Q3RAD underestimation could partially be due to the $R(Z)$ relationships being unrepresentative of the local DSDs. In addition, there were significant beam blockages in the region from both KMRX (Knoxville/Morristown, Tennessee) and KGSP (Greer, South Carolina) as shown in the radar QPE quality index (RQI; Zhang et al. 2012b) field (Fig. 15). RQI is a function of the hybrid scan beam height, blockage, and freezing level height. It decreases with increasing blockages, increasing beam heights and decreasing freezing levels. Even though the hybrid scan 


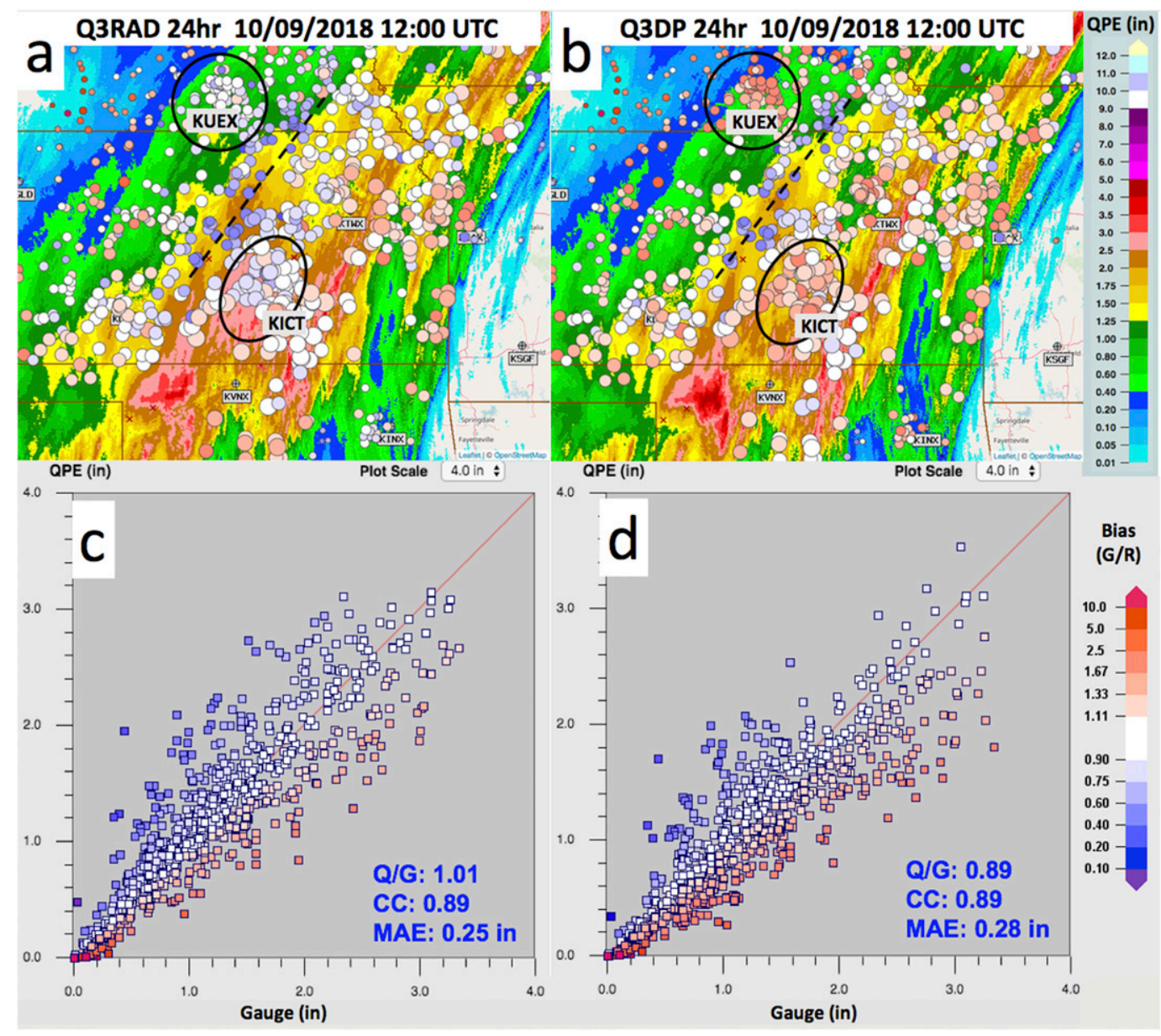

FIG. 12. As in Fig. 5, but for 24-h (a),(c) Q3RAD and (b),(d) Q3DP ending at 1200 UTC 9 Oct 2018 in Nebraska, Kansas, and Oklahoma. The black circles highlight areas with notable differences between Q3RAD and Q3DP and the dashed black line an area of overestimation associated with bright band.

reflectivity in Q3RAD was adjusted for partial beam blockages before $R(Z)$ was applied, the adjustment may not be accurate when the real-time beam propagation deviates significantly from what it was under a standard atmosphere. Thus, the Q3RAD underestimation could also be due to the partial blockages in $Z$ field. This case shows the advantage of the $R(A)$ over $R(Z)$ in areas of partial beam blockages.

Another notable difference was in a light rain (24-h accumulations $<1$ in.) area between KMRX and KHTX (Huntsville/Hytop, Alabama) where Q3DP underestimated (white dashed line, Figs. 14a,b). This is similar to the underestimation issue in the 3 October KGYX (Fig. 10a) and 9 October KUEX cases (Fig. 12a). The $R(A)$ estimates from both KHTX and KMRX used default stratiform $\alpha(0.035)$ over the rainfall period. Yet the low $Z(<35 \mathrm{~dB} Z)$ and low $Z_{\mathrm{DR}}(0-0.2 \mathrm{~dB})$ values indicated that a higher $\alpha$ should be applied based on the $\alpha-Z_{\mathrm{DR}}$ relationship shown in Wang et al. (2019). It is noteworthy that both Q3RAD and Q3DP had significant underestimation in areas of radar "gaps" with low RQI values (white circles, Figs. 14 and 15). The underestimation was likely associated with the vertical variations of reflectivity and a lack of radar observations near the ground.
Figure 16 was a wide spread stratiform rain event in the Pacific Northwest. The majority of CoCoRaHS gauges reported 1-2-in. accumulations for the 24-h period ending 1400 UTC 28 October 2018. Q3RAD had a 24\% underestimation bias and 0.27-in. MAE (Figs. 16a,d). The most significant underestimation was from areas far away from the radars (white dashed lines, Fig. 16) where the radar beam overshot the precipitation processes near the ground. Q3DP without the evaporation correction (Figs. 16b,e) showed a superior performance over Q3RAD and reduced the underestimation bias to $19 \%$ and MAE to 0.24 in. However, Q3DP with evaporation correction (Figs. 16c,f) resulted in the worst underestimation bias $(30 \%)$ and MAE (0.31 in.) among the three QPEs. The detrimental effect of the evaporation correction for this specific case was a combination of 1) radar QPE was significantly underestimating in areas far away from the radar and 2) the limitations in the evaporation correction scheme (Martinaitis et al. 2018). Mitigation of such errors in poor radar coverage area remains a challenge and requires a multisensor approach using additional in situ and remote sensing observations and atmospheric model data. 

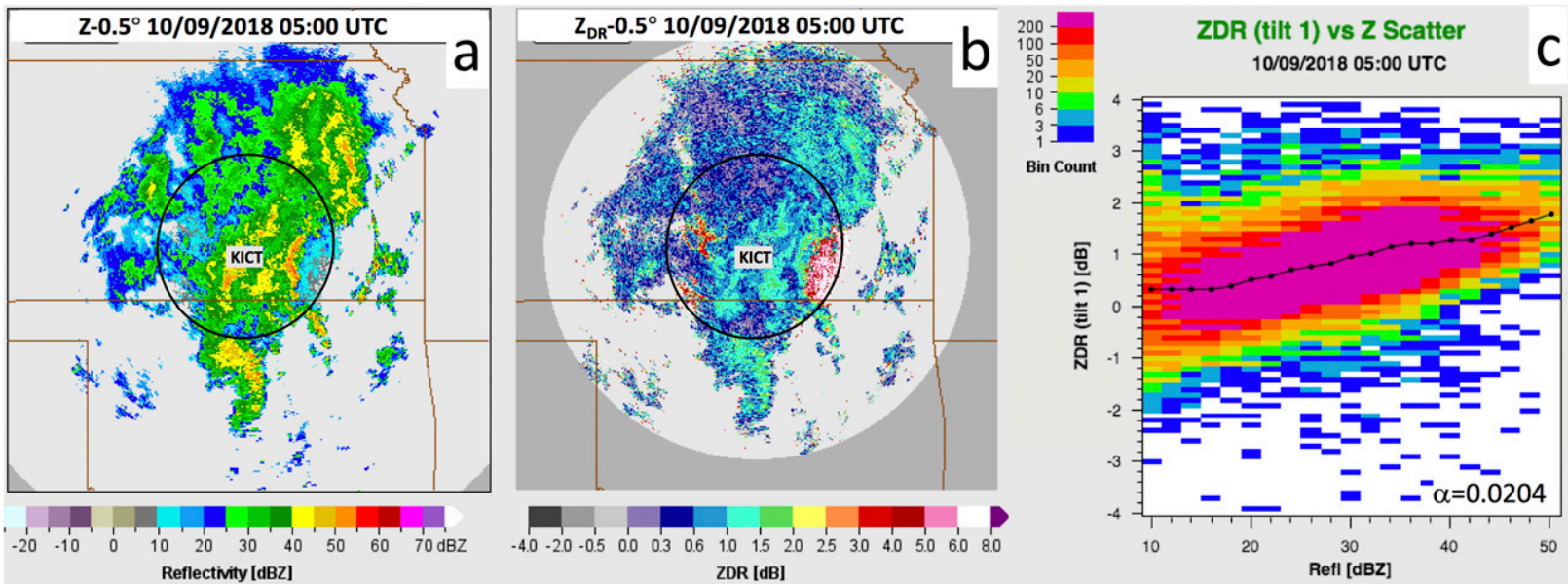

FIG. 13. As in Fig. 11, but for radar KICT at 0500 UTC 9 Oct 2018.

\section{Summary}

A new dual-polarization radar synthetic QPE (Q3DP) was developed in the MRMS system for improved accuracy of precipitation estimation. Q3DP was based on the specific attenuation $A$, the specific differential phase $K_{\mathrm{DP}}$, and reflectivity $Z$. It applies the $R(A)$ relationship in areas where radar is observing pure rain, $R\left(K_{\mathrm{DP}}\right)$ in areas with potential hail presence, and a vertical profile-corrected $R(Z)$ elsewhere.

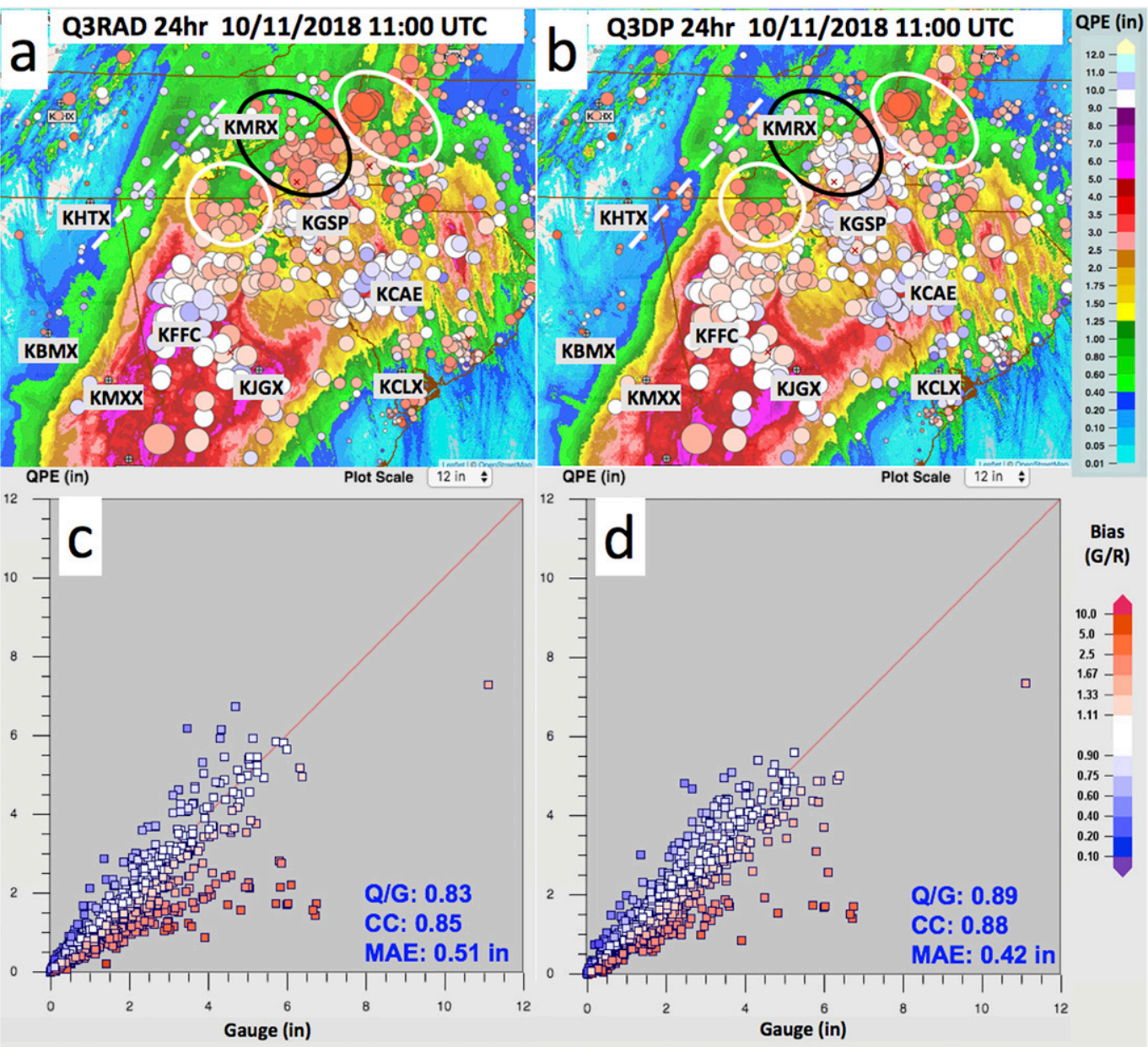

FIG. 14. As in Fig. 5, but for Georgia, South Carolina, and North Carolina at 1100 UTC 11 Oct 2018. The black circle and white dashed line highlight two areas with notable differences between Q3RAD and Q3DP. The white circles indicate areas of poor low-level radar coverage. 


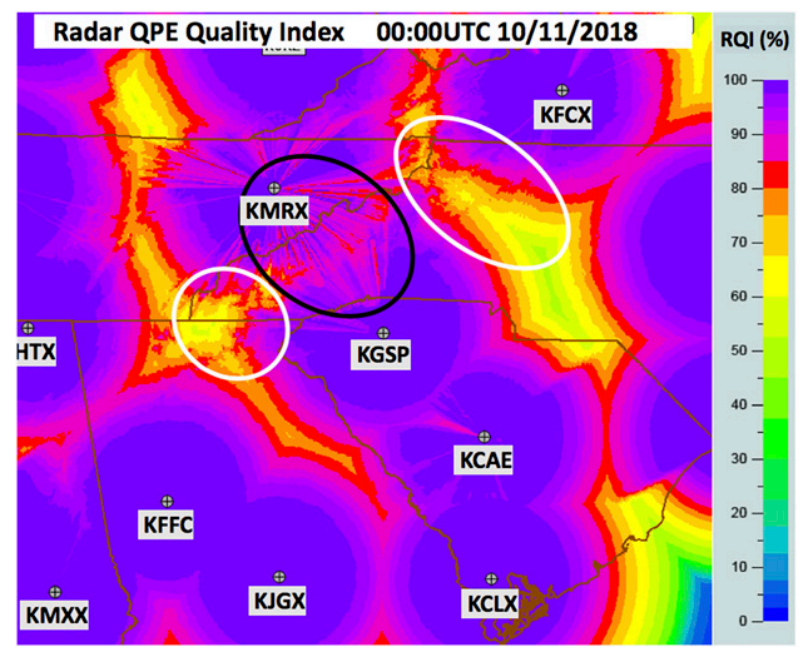

FIG. 15. Radar QPE quality index (RQI) at 0000 UTC 11 Oct 2018. The black and white circles correspond to the same circles in Figs. 14a and 14b.

The vertical profile correction is to account for large reflectivity variations in and above the melting layer. An evaporation correction based on precipitation rate, radar observation height and environmental humidity was applied to minimize false precipitation associated with subcloud evaporation.
Q3DP was evaluated across CONUS during a period of September-October 2018 and showed significant improvements over a current operational QPE based on multiple $R(Z)$. The improvements included 1) less underestimation in areas subject to partial beam blockage and in severe attenuation situations due to the insensitivity of $R(A)$ to systematic errors in $Z$ and $Z_{\mathrm{DR}}$ fields and 2) a reduction of both systematic and random errors in warm season heavy rainfall regardless of precipitation regimes and geographic locations. The improvement in 2) was attributed to a more linear relationship between $A$ and liquid water amounts than other radar variables. Another contributing factor was the dynamically updated parameter $\alpha$ in the $R(A)$ scheme that captures temporal variations of DSDs better than $R(Z)$. The extensive evaluations also indicated a few areas where Q3DP needed further refinements: $1)$ in very light and sporadic rain where the attenuation signal is too weak, $R(Z)$ may provide better estimates than $R(A)$ and should probably be applied instead of $R(A) ; 2)$ in wide spread light stratiform rain, the current default $\alpha(0.035)$ might be too low; 3) some local adjustments of $R(A)$ may be needed for more accurate estimates in precipitation of mixed regimes where the $Z-Z_{\mathrm{DR}}$ data samples are dominated by one regime. Each of these refinements are currently being tested and as appropriate will be implemented as part of the Q3DP.

Acknowledgments. The authors are grateful to Mr. Steve Martinaitis for reviewing the paper and providing valuable

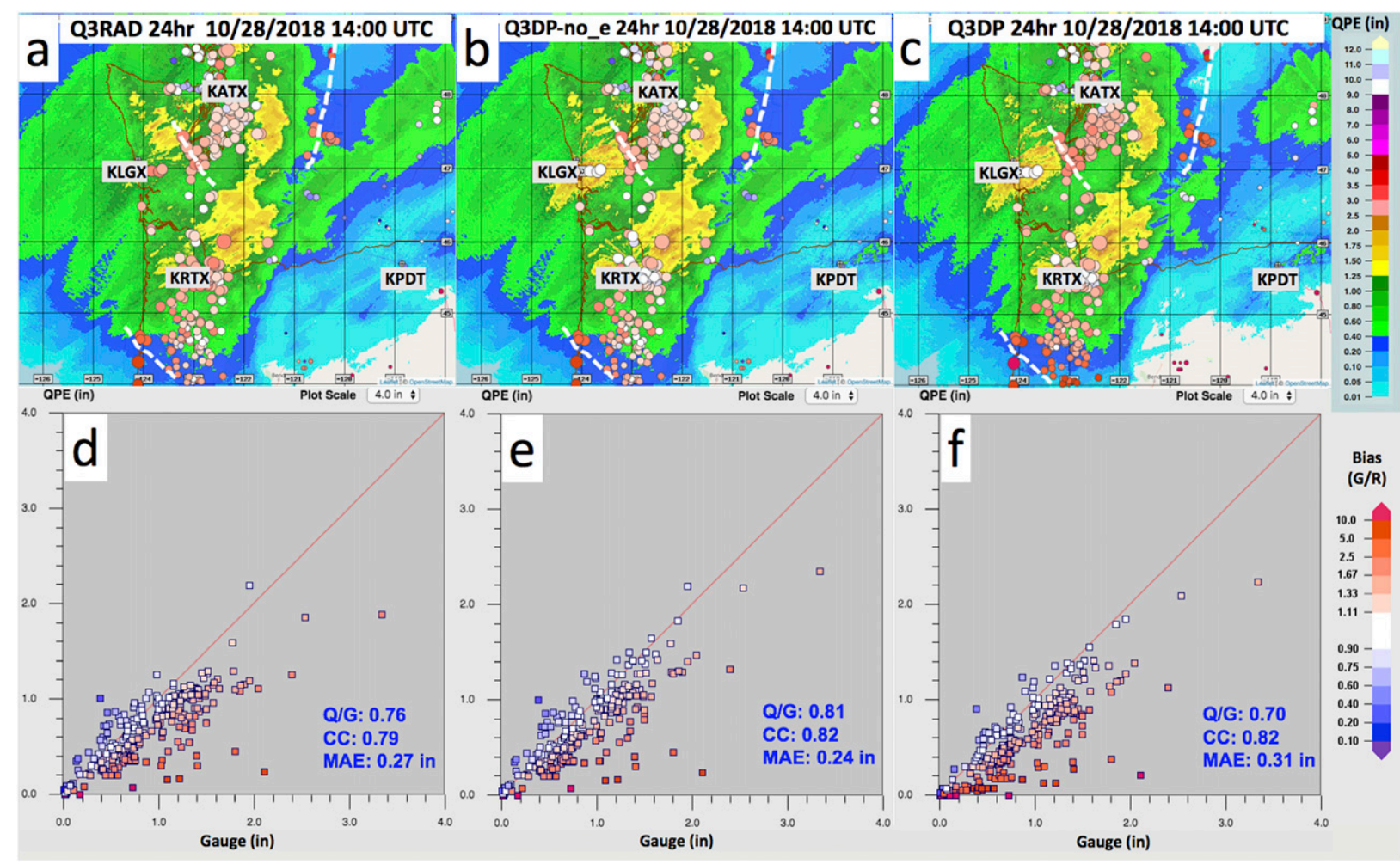

FIG. 16. As in Fig. 5, but for the Washington and Oregon area at 1400 UTC 28 Oct 2018. The white dashed lines indicate areas of poor low-level radar coverage. 
comments that greatly improved the quality of this manuscript. Dr. Heather Grams provided useful inputs through many discussions that helped refine the technique used in the paper. The work was supported by the Joint Technology Transfer Initiative (JTTI) Program within NOAA/OAR Office of Weather and Air Quality under Grant NA17OAR4590177. Additional funding was provided by NOAA/Office of Oceanic and Atmospheric Research under NOAA-University of Oklahoma Cooperative Agreement NA11OAR4320072, U.S. Department of Commerce.

\section{REFERENCES}

Cifelli, R., N. Doesken, P. Kennedy, L. D. Carey, S. A. Rutledge, C. Gimmestad, and T. Depue, 2005: The community collaborative rain, hail, and snow network: Informal education for scientists and citizens. Bull. Amer. Meteor. Soc., 86, 1069-1078, https://doi.org/10.1175/BAMS-86-8-1069.

Cocks, S. B., S. Martinaitis, B. Kaney, J. Zhang, and K. Howard, 2016: MRMS QPE performance during 2013/14 cool season. J. Hydrometeor., 17, 791-810, https://doi.org/10.1175/JHM-D15-0095.1.

—, J. Zhang, S. Martinaitis, Y. Qi, B. Kaney, and K. Howard, 2017: MRMS QPE performance east of the Rockies during the 2014 warm season. J. Hydrometeor., 18, 761-775, https:// doi.org/10.1175/JHM-D-16-0179.1.

— , and Coauthors, 2019: A prototype quantitative precipitation estimation algorithm for operational S-Band polarimetric radar utilizing specific attenuation and specific differential phase. Part II: Performance verification and case study analysis. J. Hydrometeor., 20, 999-1014, https://doi.org/10.1175/JHM-D-18-0070.1.

Fulton, R., J. Breidenbach, D.-J. Seo, D. Miller, and T. O'Bannon, 1998: The WSR-88D rainfall algorithm. Wea. Forecasting, 13, 377-395, https://doi.org/10.1175/1520-0434(1998)013<0377: TWRA $>2.0 . \mathrm{CO} ; 2$.

Giangrande, S. E., and A. V. Ryzhkov, 2008: Estimation of rainfall based on the results of polarimetric echo classification. J. Appl. Meteor. Climatol., 47, 2445-2462, https:// doi.org/10.1175/2008JAMC1753.1.

Martinaitis, S. M., H. M. Grams, C. Langston, J. Zhang, and K. Howard, 2018: A real-time evaporation correction scheme for radarderived mosaicked precipitation estimations. J. Hydrometeor., 19, 87-111, https://doi.org/10.1175/JHM-D-17-0093.1.

Park, H. S., A. V. Ryzhkov, D. S. Zrnić, and K.-E. Kim, 2009: The hydrometeor classification algorithm for the polarimetric WSR-88D: Description and application to an MCS. Wea. Forecasting, 24, 730-748, https://doi.org/10.1175/2008WAF2222205.1.
Qi, Y., and J. Zhang, 2017: A physically based two-dimensional seamless reflectivity mosaic for radar QPE in the MRMS system. J. Hydrometeor., 18, 1327-1340, https://doi.org/10.1175/JHM-D16-0197.1.

Ryzhkov, A. V., and D. Zrnić, 2019: Radar Polarimetry for Weather Observations. Springer, 486 pp.

- , S. E. Giangrande, and T. J. Schuur, 2005: Rainfall estimation with a polarimetric prototype of WSR-88D. J. Appl. Meteor., 44, 502-515, https://doi.org/10.1175/JAM2213.1.

— M. Diederich, P. Zhang, and C. Simmer, 2014: Potential utilization of specific attenuation for rainfall estimation, mitigation of partial beam blockage, and radar networking. J. Atmos. Oceanic Technol., 31, 599-619, https://doi.org/10.1175/ JTECH-D-13-00038.1.

Tang, L., J. Zhang, C. Langston, J. Krause, K. Howard, and V. Lakshmanan, 2014: A physically based precipitationnonprecipitation radar echo classifier using polarimetric radar and environmental data in a real-time system. Wea. Forecasting, 29, 1106-1119, https://doi.org/10.1175/WAF-D13-00072.1.

Wang, Y., P. Zhang, A. Ryzhkov, J. Zhang, and P.-L. Chang, 2014: Utilization of specific attenuation for tropical rainfall estimation in complex terrain. J. Hydrometeor., 15, 2250-2266, https://doi.org/10.1175/JHM-D-14-0003.1.

- , S. Cocks, L. Tang, A. Ryzhkov, P. Zhang, J. Zhang, and K. Howard, 2019: A prototype quantitative precipitation estimation algorithm for operational S-band polarimetric radar utilizing specific attenuation and specific differential phase. Part I: Algorithm description. J. Hydrometeor., 20, 985-997, https://doi.org/10.1175/JHM-D-18-0071.1.

Zhang, J., and Y. Qi, 2010: A real-time algorithm for the correction of brightband effects in radar-derived QPE. J. Hydrometeor., 11, 1157-1171, https://doi.org/10.1175/2010JHM1201.1.

$\longrightarrow, \ldots$, D. Kingsmill, and K. Howard, 2012a: Radar-based quantitative precipitation estimation for the cool season in complex terrain: Case studies from the NOAA hydrometeorology testbed. J. Hydrometeor., 13, 1836-1854, https://doi.org/ 10.1175/JHM-D-11-0145.1.

,,-- K. Howard, C. Langston, and B. Kaney, 2012b: Radar Quality Index (RQI) - A combined measure of beam blockage and VPR effects in a national network. IAHS Publ., 351, 388-393.

, and Coauthors, 2016: Multi-Radar Multi-Sensor (MRMS) quantitative precipitation estimation: Initial operating capabilities. Bull. Amer. Meteor. Soc., 97, 621-638, https://doi.org/ 10.1175/BAMS-D-14-00174.1. 